\title{
La modélisation numérique fine appliquée à l'analyse thermohydraulique *
}

\author{
P. L. Viollet ${ }^{\mathrm{a}}$, D. Grand ${ }^{\mathrm{b}}$, J.-P. Chabard ${ }^{\mathrm{a}}$ et J.-P. Magnaud ${ }^{\mathrm{c}}$ \\ ${ }^{a}$ Electricité de France, Direction des Etudes et Recherches, Laboratoire National d'Hydraulique, \\ 6 Quai Watier, 78400 Chatou, France. \\ b Commissariat à l'Energie Atomique, Direction des Réacteurs Nucléaires, Département de \\ Thermohydraulique et de Physique, 17, rue des Martyrs, 38054 Grenoble Cedex 9, France. \\ c Commissariat à l'Energie Atomique, Direction des Réacteurs Nucléaires, \\ Département de Mécanique et de Technologie, 91191 Gif-sur-Yvette Cedex, France.
}

\section{I $\square$ INTRODUCTION}

La thermohydraulique ${ }^{1}$ monophasique joue un rôle important dans la conception des réacteurs nucléaires. Le fluide de refroidissement est en phase liquide pendant la majeure partie des conditions de fonctionnement d'un réacteur, tout au long de sa durée de vie et quel qu'en soit le type, réacteur à eau sous pression ou réacteur à neutrons rapides refroidi au métal liquide.

La température relativement élevée ainsi que les grandes différences de température dans le fluide peuvent être à l'origine de contraintes thermiques dans les matériaux du réacteur qui viennent s'ajouter aux contraintes mécaniques déjà présentes. Il y a donc lieu de tenir compte de ces contraintes thermiques lors de la conception. A cet égard, le rôle de l'analyse thermohydraulique est de fournir aux concepteurs de réacteurs des résultats ou des outils leur permettant de prédire la sollicitation thermique des structures.

* Cet article est déjà paru en anglais dans Nuclear Engineering and design 124, (1990), p. 339-361.
Dans les réacteurs à neutrons rapides, l'existence de grands volumes de fluide de refroidissement (sodium) dans le circuit primaire se prête à l'apparition de zones stratifiées, dans lesquelles la température du fluide de refroidissement varie sur de courtes distances le long de l'axe vertical. Un phénomène comparable peut apparaître dans les conduites de grande section lorsque la vitesse débitante est assez petite pour que les forces de flottabilité soient égales ou supérieures aux forces d'inertie. Ceci peut se produire dans les conduites du circuit primaire des réacteurs à eau sous pression (REP) ou dans les circuits secondaires des réacteurs à neutrons rapides refroidis au métal liquide.

Le présent article traite de la résolution de ces problèmes de thermohydraulique monophasique. Les résultats présentés proviennent de réalisations expérimentales ou de simulations numériques. Ces simulations numériques ont été réalisées à l'aide de logiciels développés au CEA et à EDF pour prédire les écoulements incompressibles multidimensionnels couplés au champ de température par des forces de flottabilité. Le but de la présente étude est également de fournir des informations générales sur les développements sur lesquels se fondent ces codes.

\section{Refined numerical modelling in thermal-hydraulic analysis}

The paper presents the strategy for the development of numerical models and software in EDF and CEA, for the computation of nuclear reactor single-phase thermal-hydraulics problems. These tools use the Navier-Stokes equations coupled with the $k-\varepsilon$ model for turbulence, and have been validated upon simple situations of isothermal and non-isothermal flows, including recirculating flows, and stratified flows appearing under transient situations in closed cavities or in pipes. Further developments of these tools are undertaken in order to reach better accuracy in the treatment of some particularly complex turbulent flow situations, and in order to get informations on the temperature fluctuations. In that scope, use was made of transport equations for Reynolds stresses, turbulent heat fluxes, and/or for the dissipation rate of temperature fluctuations. Results with these second moment closures are shown on stratified channel flow, heated boudary layers and jets. 
Depuis une dizaine d'années, on a tendance à utiliser de plus en plus les simulations numériques. Cette tendance est générale, néanmoins il peut être intéressant d'en trouver les raisons sous-jacentes.

\subsection{Fourniture de données pour un programme de ther- momécanique}

L'un des objectifs des études de thermohydraulique est de fournir des informations sur l'analyse thermomécanique des structures, concernant la charge dynamique (pression, vitesse) ou thermique exercée par le fluide sur ces structures. L'analyse thermomécanique est conduite avec des modèles numériques multidimensionnels qui nécessitent la distribution de paramètres physiques (température,...) à l'interface entre le fluide et les structures. La transmission de nombreuses données aux programmes de thermomécanique justifie un système comparable à celui qui existe dans le domaine de la thermohydraulique.

\subsection{Limites des modèles physiques}

Il y a quinze ans, les modèles physiques étaient encore les seules sources de données dans le domaine de la thermohydraulique, en raison des difficultés rencontrées dans l'élaboration de modèles numériques dans ce domaine. Ainsi, les modèles physiques ont été, et sont encore, très utilisés dans les simulations thermohydrauliques.

Cependant, les modèles physiques ne permettent pas de tenir compte de toute la complexité des situations à simuler.

Premièrement, un modèle physique à petite échelle ne peut satisfaire à tous les critères de similitude nécessaires. Dans un écoulement monophasique, en l'absence de surface libre, et dans une situation régulière, un écoulement avec convection mixte est caractérisé par trois nombres adimensionnels, à savoir le nombre de Péclet, le nombre de Reynolds et le nombre de Richardson (ou nombre de Froude densimétrique). Or, ces trois nombres ne peuvent être obtenus simultanément avec un modèle physique d'un prototype. II faut tenir compte de certaines distorsions. II faut donc procéder à des extrapolations par le biais de l'analyse et de l'expérience acquise, pour déterminer les débits et les quantités de chaleur applicables au prototype.

Deuxièmement, il est difficle de produire certaines conditions aux limites dans un modèle physique. C'est le cas des conditions aux limites thermiques imposées aux limites externes de l'écoulement: distribution du flux de chaleur ou condition d'échange.

\subsection{Améliorations des modèles numériques}

L'élaboration d'algorithmes numériques appropriés pour résoudre les équations de Navier-Stockes et l'obtention de modèles de turbulence satisfaisants bien qu'approximatifs ont transformé la situation depuis quinze ans. En outre, l'augmentation de la capacité de mémoire des ordinateurs permet de résoudre des problèmes d'écoulement en 2D et en 3D avec la discrétisation appropriée. A cet égard, le lecteur se reportera utilement à des articles tels que $[4,5]$.
Le but de cet article est de présenter les développements de codes de calcul réalisés au CEA et à EDF. Ces codes tridimensionnels sont :

- TRIO, qui comporte un solveur en volumes finis et un solveur en éléments finis (TRIO VF et TRIO EF), du CEA : - ESTET, qui est un code en volumes finis, et N3S qui est un code en éléments finis, d'EDF.

Le présent article porte délibérément sur le développement des modélisations physiques sous-jacentes à ces codes ainsi que sur leur validation sur la base de résultats d'expériences. C'est pourquoi les méthodes numériques utilisées dans les codes ne sont que brièvement décrites dans les annexes de cet article.

La première partie traite de la modélisation de la turbulence avec un modèle à deux équations (le modèle $k-\varepsilon$ bien connu). Les validations données pour ce modèle concernent différents types d'écoulements typiques de ceux que l'on rencontre dans des réacteurs nucléaires : écoulements isothermes avec recirculations, stratifications thermiques dans des liquides. Cette présentation tentera de répondre aux questions suivantes : dans quelle mesure ce modèle peut-il être appliqué à un réacteur et quelle précision peut-on en attendre ?

Dans une deuxième partie, des modèles plus perfectionnés sont présentés qui portent sur les moments statistiques du second ordre (tensions de Reynolds et flux turbulent de quantité de chaleur). La raison de cette complexité supplémentaire de la modélisation sera expliquée surtout par le besoin de résultats sur la partie fluctuante du champ de température.

\section{VALIDATION DES MODÈLES NUMÉRI- QUES ET APPLICATION À L'ANALYSE THERMOHYDRAULIQUE}

\subsection{Les équations de base}

La présente étude s'intéresse aux écoulements monophasiques rencontrés, par exemple, dans les circuits primaires des réacteurs à eau sous pression (REP) ou dans les circuits primaires et secondaires des réacteurs à neutrons rapides refroidis au métal liquide. Ces écoulements sont des écoulements internes turbulents. Les différences de température peuvent être relativement grandes (jusqu'à $200^{\circ} \mathrm{C}$ ), et les effets de flottabilité liés à ces différences de température peuvent être importants. Les écoulements considérés ici sont des écoulements liquides et les différences de densité relative liées à ces différences de température restent généralement faibles. On peut donc écrire la densité comme étant la somme d'une densité de référence $\rho_{0}$ (qui peut être soit une constante, soit fonction de la hauteur) et d'une variation de densité $\rho^{*}$ résultant des variations de température, en supposant que :

$$
\rho * / \rho_{0} \ll 1 \quad\left(\rho=\rho_{0}+\rho^{*}\right)
$$

Cela permet de linéariser le terme de gradient de pression dans les équations de Navier-Stokes :

$$
-\frac{1}{\rho} \overrightarrow{\operatorname{grad}} p+\vec{g}=-\frac{1}{\rho_{0}} \overrightarrow{\operatorname{grad}} p^{*}+\frac{\rho^{*}}{\rho_{0}} \vec{g},
$$


où $p^{*}$ est la différence de pression par rapport à la distribution de pression hydrostatique liée à la distribution de densité $\rho_{0}$. C'est ce qu'on appelle l'approximation de Boussinesq, qui permet aussi, dans de nombreuses applications, de considérer comme négligeables les différences de densité dans l'équation de continuité (conservation de la masse). Le système de base d'équations nécessaires à un calcul thermohydraulique apparaît ainsi, si on l'écrit selon un système de coordonnées cartésiennes $x_{i}, i=1$ à 3 .

Conservation de la masse moyennée :

$$
\partial \bar{u}_{i} / \partial x_{i}=0 .
$$

Équation de Reynolds :

$$
\frac{\partial \bar{u}_{i}}{\partial t}+\bar{u}_{j} \frac{\partial \bar{u}_{i}}{\partial x_{j}}=-\frac{1}{\rho_{0}} \frac{\partial \overline{p^{*}}}{\partial x_{i}}+\frac{\partial}{\partial x_{j}}\left(-\overline{u_{i}^{\prime} u_{j}^{\prime}}+v \frac{\partial \bar{u}_{i}}{\partial x_{j}}\right)+\overline{\rho^{*}} \frac{\overline{\rho_{0}}}{g i} .
$$

Bilan énergétique moyenné :

$$
\frac{\partial \bar{T}}{\partial t}+\bar{u}_{j} \frac{\partial \bar{T}}{\partial x_{j}}=\frac{\partial}{\partial x_{j}}\left(-\overline{u_{j}^{\prime} T^{\prime}}+\kappa \frac{\bar{T}}{\partial x_{j}}\right) .
$$

Ces équations font intervenir un opérateur de moyenne, défini selon la méthode classique des moyennes de Reynolds (les moyennes sont notées avec une barre horizontale, et toute fluctuation d'un paramètre $G$ s'écrit $G^{\prime}$ ).

Les inconnues naturelles qui apparaissent dans ces équations de base sont les tensions de Reynolds $R_{i j}$ et les flux turbulents $R_{i l}$ définis comme :

$$
R_{i j}=\overline{u_{i}^{\prime} u_{j}^{\prime}} \text { et } R_{i t}=\overline{u_{j}^{\prime} T^{\prime}} .
$$

Pendant de nombreuses années, l'utilisation des modèles aux tensions de Reynolds consistant à résoudre des équations de transport modélisées pour $R_{i j}$ et $R_{i t}$ a été jugée trop difficile pour les calculs elliptiques d'écoulements multidimensionnels. Pendant longtemps, l'hypothèse de viscosité turbulente, avec le modèle $k-\varepsilon$ [1], est apparue comme un bon compromis [2]: dans ce modèle, utilisé dans tous les calculs présentés dans ce chapitre, deux équations modélisées seulement sont résolues, l'une pour l'énergie cinétique turbulente :

$k=\frac{1}{2} \overline{u_{i}^{\prime} u_{j}^{\prime}}$ et l'autre pour son taux de dissipation $\varepsilon$.

Le modèle $k-\varepsilon$ se fonde sur deux hypothèses principales :

(a) le tenseur des tensions de Reynolds est supposé colinéaire avec le tenseur des contraintes moyennes (viscosité turbulente), et le flux turbulent de quantité de chaleur est supposé colinéaire avec le gradient de température moyen (diffusivité turbulente) ;

(b) la turbulence est supposée être en équilibre spectral, la turbulence n'étant produite qu'à une échelle de longueur, et pour un transfert d'énergie $\varepsilon$ constant dans la gamme inertielle.

L'expression des écoulements turbulents découle de (a) :

$$
R_{i j}=\overline{u_{i}^{\prime} u_{j}^{\prime}}=-v_{t}\left(\frac{\partial \bar{u}_{i}}{\partial x_{j}}+\frac{\partial \bar{u}_{j}}{\partial x_{i}}\right)+\frac{2}{3} k \delta_{i j}
$$

$$
R_{i t}=\overline{u_{j}^{\prime} T^{\prime}}=-\kappa \partial \bar{T} / \partial x_{j}
$$

L'expression de Prandtl-Kolmogorov pour la viscosité turbulente et la diffusivité turbulente découle de (b) :

$$
\begin{aligned}
& v_{t}=C_{\mu} k^{2} / \varepsilon \\
& \kappa_{t}=\frac{C_{\mu}}{\sigma_{t}} \frac{k^{2}}{\varepsilon}
\end{aligned}
$$

où $C_{\mu}$, et le nombre de Prandtl turbulent $\sigma_{r}$, sont considérés comme des constantes.

D'après Launder et Spalding [1], les équations de transport pour $k$ et $\varepsilon$ s'écrivent :

$$
\begin{aligned}
\frac{\partial k}{\partial t}+\bar{u}_{k} \frac{\partial k}{\partial x_{k}} & =\frac{\partial}{\partial x_{k}}\left(\frac{v_{t}}{\sigma_{k}} \frac{\partial k}{\partial x_{k}}\right)+\dot{P}+\bar{G}-\varepsilon \\
\frac{\partial \varepsilon}{\partial t}+\bar{u}_{k} \frac{\partial \varepsilon}{\partial x_{k}} & =\frac{\partial}{\partial x_{k}}\left(\frac{v_{t}}{\sigma_{\varepsilon}} \frac{\partial \varepsilon}{\partial x_{k}}\right)+ \\
C_{\varepsilon 1} \frac{\varepsilon}{k} & {\left[\dot{P}+\left(1-C_{\varepsilon 3}\right) \tilde{G}\right]-C_{\varepsilon 2} \frac{\varepsilon^{2}}{k} }
\end{aligned}
$$

$\tilde{P}$ est le taux de production:

$$
\dot{P}=-\overline{u_{i}^{\prime} u_{j}^{\prime}} \frac{\partial \bar{u}_{i}}{\partial x_{j}}
$$

et $\tilde{G}$ est la contribution de l'effet de flottabilité :

$$
\tilde{G}=-3 g_{i} \overline{u_{i}^{\prime} T^{\prime}} .
$$

Le choix de $C_{\varepsilon 3}$ constant (lié à l'effet de flottabilité dans l'équation de dissipation) a été largement discuté : Gibson et Launder [3] ont utilisé la valeur 1 dans un écoulement stratifié de façon stable, alors que Rodi [4] a proposé la valeur 0,8 et plus récemment la valeur 1 pour des écoulements stratifiés de façon stable. Viollet $[4,5]$ a proposé le choix suivant :

$C_{\varepsilon 3}=1$ dans un écoulement stratifié de façon stable $(\bar{G}<0)$;

$C_{\varepsilon 3}=0$ dans un écoulement stratifié de façon instable $(\bar{G}<0)$.

Ce choix a été bien confirmé par les simulations de grands tourbillons réalisées par Khoudli [6], et, sans autre mention, sera repris dans les calculs rapportés ici.

Le nombre de Prandtl turbulent $\sigma_{t}$ est pris entre 0,8 et 1 (à l'intérieur de cette plage, on considère qu'une variation n'a généralement guère d'influence), alors que toutes les autres constantes sont empruntées à Launder et Spalding [1].

2.2 Présentation des études de validation rapportées aux $\S 2.3$ à 2.5

Le degré de confiance accordé aux résultats fournis par un modèle numérique dépend de la plage à l'intérieur de laquelle le code a été validé. Au début de la validation des 


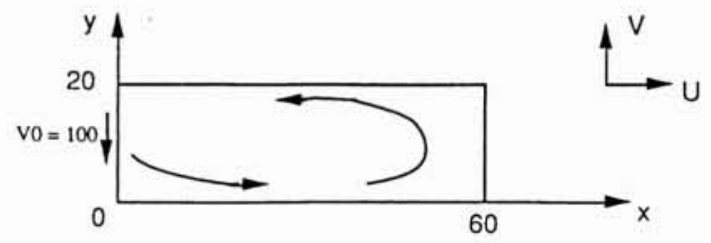

1. Géométrie de la cavité à paroi mobile dans l'expérience [10] (unités en $\mathrm{cm}$, s).

codes de calculs d'écoulements turbulents, des cas-tests simples ont été retenus de façon à mettre en évidence un ou plusieurs phénomènes physiques particuliers. Cela devrait permettre de répondre plus facilement aux questions : dans quelle mesure le code permet-il de reproduire un écoulement avec recirculations (ou un jet turbulent ou un écoulement stratifié ou un transfert de chaleur à la paroi) ? A cet effet, des cas-tests simples ont été étudiés en premier. Certains ont été choisis pour être étudiés comme cas-tests par le groupe de travail sur la modélisation fine des écoulements de $\mathrm{l}^{1} \mathrm{AIRH}^{2}$ : ce groupe de travail est parvenu à des solutions $k-\varepsilon$ ralliant un consensus et qui ont été comparées à des expériences portant sur un élargissement brusque axisymétrique [7, 8], une cavité à paroi mobile [9], des écoulements périodiques sur des obstacles, des écoulements stratifiés en canal,...

Le présent article détaille la validation des codes en présence d'écoulements avec recirculations et de phénomènes de stratification. Le \$ 2-3 donne un exemple type d'un cas-test concernant un écoulement isotherme avec recirculations. Le $\$ 2-4$ montre les résultats d'une validation sur une stratification dans une cavité remplie de sodium et résultant d'un changement de la température d'entrée. Le \$2-5 termine ce chapitre avec un type d'écoulement particulièrement intéressant dans une étude de réacteur: les courants de densité et les écoulements stratifiés que l'on peut rencontrer dans des conduites dans des situations de faible débit.

\section{3 Écoulement isotherme turbulent dans une cavité à paroi mobile}

L'écoulement dans une cavité rectangulaire à paroi mobile est un cas-test classique de calcul d'un écoulement laminaire en deux ou trois dimensions. Il a été possible d'étendre ce cas-test à des calculs d'écoulements turbulents grâce à la réalisation par Normandin [10] d'une étude expérimentale dans cette configuration.

Cette expérience a été choisie par le groupe de travail de l'AIRH pour un problème test [9] portant sur la géométrie 2D illustrée à la figure 1 . La paroi mobile est le petit côté du rectangle en $x=0$, sa vitesse de défilement est $V=-100 \mathrm{~cm} / \mathrm{s}$. Les propriétés physiques du fluide sont $\sigma=1$ et $\mu=10^{-2}$ cgs. Dans cette expérience, plusieurs écoulements 3D ont été observés dans le sens vertical. Toutefois, ils étaient assez faibles pour que le plan à mi-hauteur de la cavité soit représentatif d'un écoulement 2D. La vitesse moyenne, l'énergie cinétique turbulente et

(2) Association Internationale pour la Recherche en Hydraulique, Division des Phénomènes Fluides dans la Production d'énergie les tensions de Reynolds ont été mesurées dans ce plan et peuvent être comparées aux prédictions du code.

La référence [9] rapporte dans leur totalité les résultats de 11 contributions. Parmi ces résultats, nous avons sélectionné ceux qui ont été obtenus sur une grille prescrite. Le but de cette grille était de faire en sorte que les erreurs numériques soient de grandeur comparable et que les différences entre les prédictions dépendent uniquement des modèles de turbulence.

Le modèle $k-\varepsilon$ a été utilisé dans les contributions suivantes :

\section{- Meyer avec TRIO-VF \\ - Viollet (1) avec ESTET \\ - Wilkes.}

Dans ces trois cas, on a supposé un profil de vitesse logarithmique et un équilibre local pour la turbulence de façon à calculer l'écoulement dans les couches limites à proximité des parois.

Une quatrième contribution de Viollet (2) s'est fondée sur un traitement de la paroi avec des résolutions numériques unidimensionnelles dans la couche limite en utilisant un modèle de turbulence à bas nombre de Reynolds.

Les résultats des calculs et leur comparaison avec l'expérience sont présentés à la figure 2 pour une section transversale placée en $x=28$. Les prédictions de la vitesse moyenne sont très proches les unes des autres et correspondent bien aux résultats de l'expérience. Cependant, l'énergie cinétique turbulente et, par voie de conséquence, les tensions de Reynolds, sont sous-estimées dans les prédictions faites par les codes. Cette tendance se trouve confirmée par les résultats obtenus sur d'autres sections tel qu'indiqué dans [9] et non reproduits ici. Dans tous les cas, les prédictions de vitesse moyenne correspondent très bien aux résultats de l'expérience. Les quantités turbulentes sont toujours sous-estimées par les prédictions du code, les divergences augmentant pour des valeurs de plus en plus grandes sur l'abscisse $x$ de la section. Le calcul a également été effectué par Chabard avec le code N3S, avec une grille légèrement différente et des résultats similaires.

\subsection{Modélisation des écoulements stratifiés dans des ca- vités fermées : une expérience en sodium}

Dans les réacteurs nucléaires, les différences de température peuvent être à l'origine d'effets de flottabilité et de stratifications thermiques. L'interaction d'un écoulement recirculant avec une stratification stable a été étudiée dans un cas de géométrie simple : une cavité rectangulaire dont la partie inférieure communique avec une canalisation. Une convection forcée est imposée dans la canalisation; elle entraîne une recirculation de l'écoulement dans la cavité. Une diminution progressive de la température d'admission provoque une stratification thermique.

Un important programme expérimental et analytique a été appliqué à ce problème, dont la géométrie est illustrée à la figure 3. Divers tests concernant le sodium sont rapportés par Vidil et coll. [11]. Ils fournissent une base de données pour la validation du code.

La cavité a une hauteur de $3,2 \mathrm{~m}$ de façon à donner des échelles de longueur et des valeurs des nombres adimensionnels comparables aux valeurs rencontrées sur un réacteur. Au cours d'un test, une recirculation isotherme est prise comme condition initiale. Une diminution de la tem- 

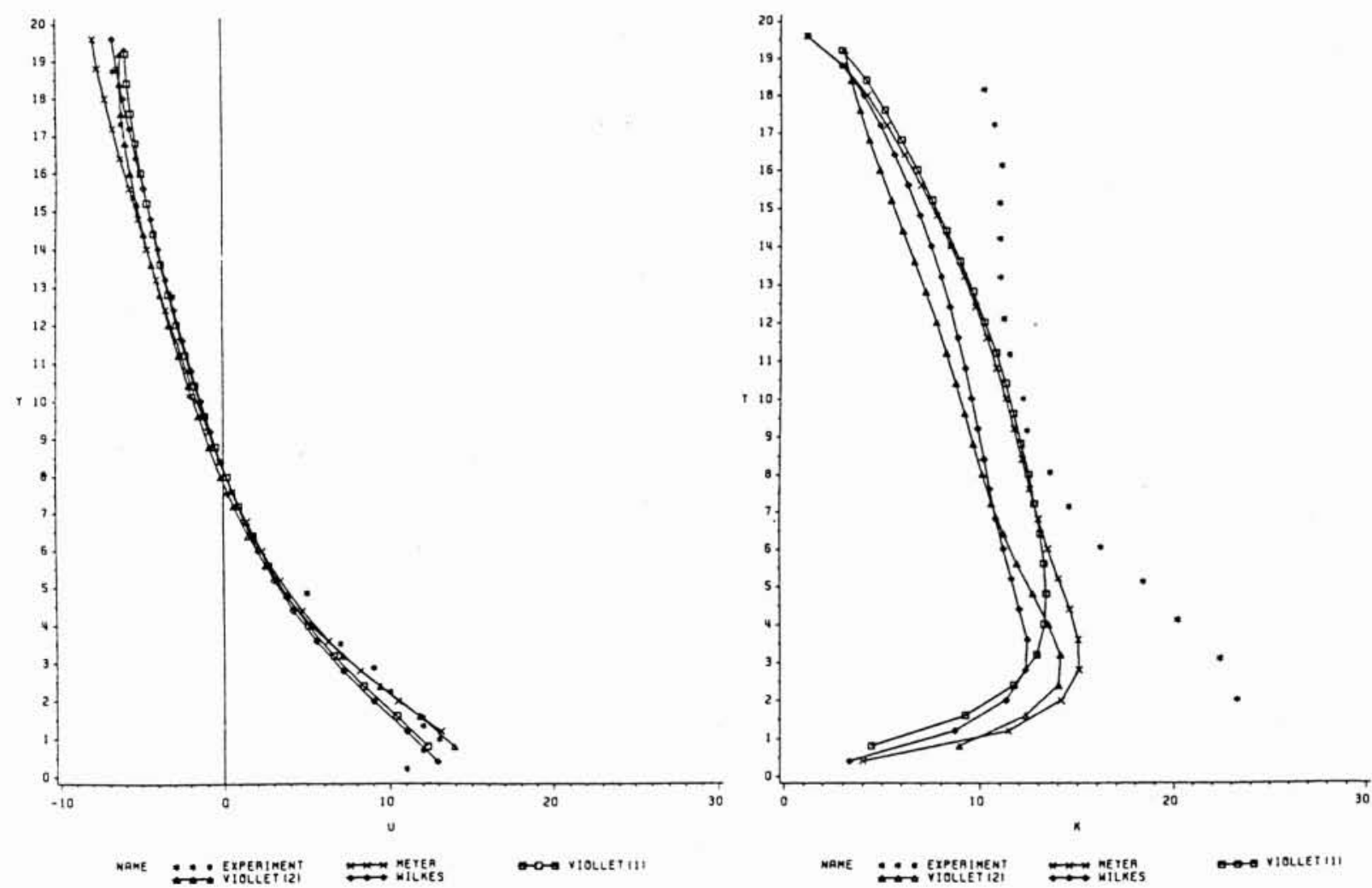

2. Distribution transversale de la vitesse moyenne $U$ (gauche) et de l'énergie cinétique turbulente $k$ (droite) à $x=28$ (source : Wilkes [9]).

pérature d'admission déclenche alors un régime thermique transitoire dans la cavité qui peut aboutir à une stratification stable dans la cavité si le nombre de Richardson est suffisamment élevé.

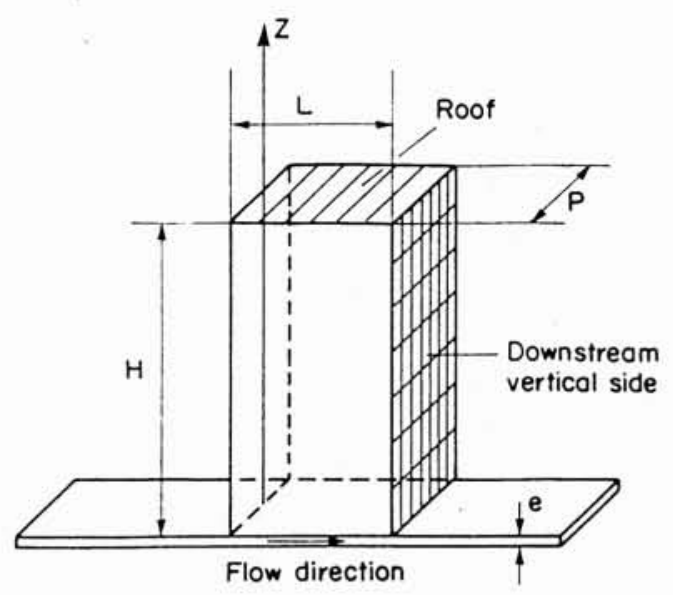

3. Description de la cavité et de l'instrumentation correspondant à l'expérience d'écoulement stratifié de sodium en régime transitoire (source: Vidil et coll. [11]).
Le nombre de Richardson global est donné par:

$$
R_{i}=g \beta \delta T H / U^{2},
$$

où $\delta T$ est la chute de température au cours du régime transitoire, $H$ est la hauteur de la cavité et $U$ la vitesse d'entrée. Dans ce cas, les évènements suivants se produisent successivement :

- Un effet de « clapotis » au cours de la phase initiale du régime transitoire : le fluide entrant plus froid monte dans un premier temps sous l'effet de la recirculation initiale, puis retombe sous l'influence des forces de flottabilité. Un certain volume du fluide initialement chaud reste dans la partie supérieure de la cavité. L'interface entre le fluide chaud et le fluide froid est soumise à des oscillations qui s'amortissent. La position initiale de cette interface dépend des paramètres-clés suivants : les nombres de Richardson (ou nombre de Froude densimétrique) et nombre de Péclet.

- Une fois que l'interface est horizontale, on observe une lente montée de celle-ci, provoquée par le transfert de chaleur de la région chaude supérieure vers la recirculation.

On a comparé à ces résultats ceux fournis par les codes developpés par le CEA et EDF et qui utilisent l'un comme l'autre le modèle de turbulence $k$ - $\varepsilon$, et on a démontré qu'ils permettaient de prédire la stratification thermique, l'ampleur du gradient de température et l'évolution transitoire de la stratification (Fig. 4). La référence [12] donne plus de détails sur ces comparaisons. 
z

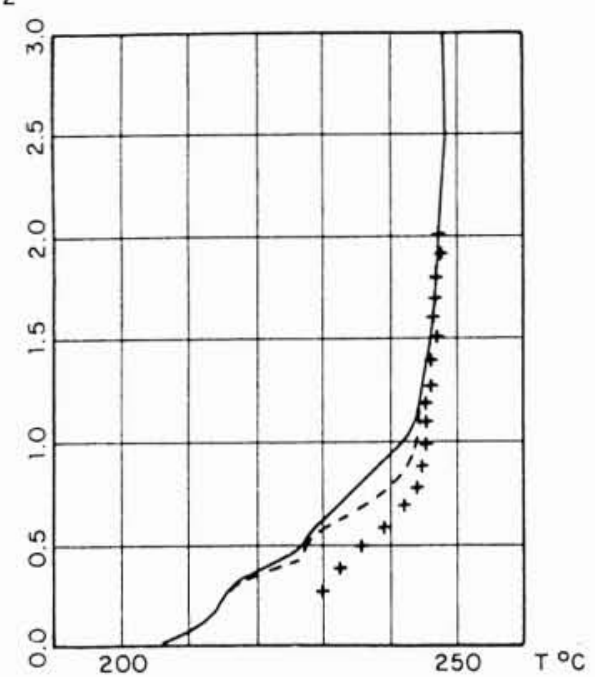

z

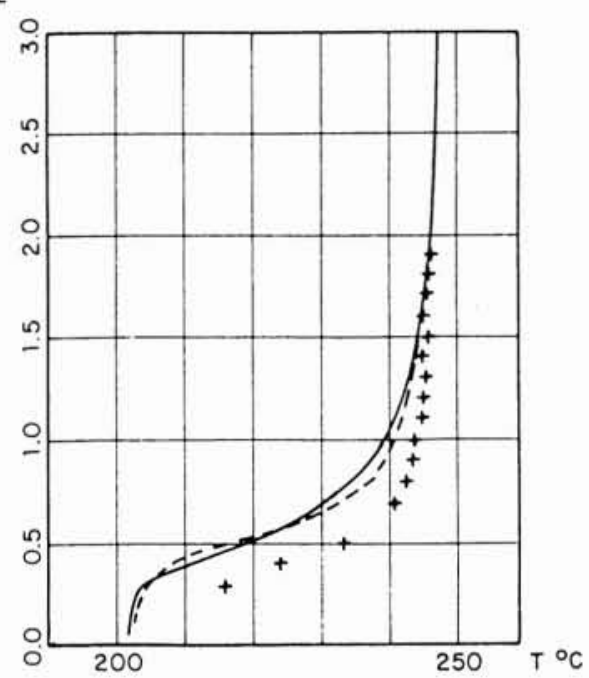

4. Profils de température verticale calculés et mesurés sur l'axe de la cavité à $420 \mathrm{~s}$ et $600 \mathrm{~s}$ après la diminution de la température d'entrée dans le canal (source: Grand et coll. [12]) (+ représente les résultats de l'expérience ; les traits pleins et les pointillés représentent les résultats des calculs).

\subsection{Stratifications et effets de densité dans les conduites}

\subsubsection{Brève analyse des conséquences des effets de densité} dans les conduites

Tous les réacteurs nucléaires ont des réseaux de canalisation relativement longs. Dans certaines conditions de fonctionnement, on peut rencontrer des situations dans lesquelles la vitesse $V$ dans la conduite (diamètre $D$ ) est petite, et où la différence de densité $\left(\rho_{1}-\rho_{2}\right)$ entre deux extrêmités de la conduite est relativement grande.

En voici quelques exemples :

- Un phénomène transitoire dans un circuit secondaire d'un réacteur à neutrons rapides refroidi au métal liquide, avec une petite vitesse $V$ et une densité variant de $\rho_{1}$ (valeur initiale) à $\rho_{2}$ (valeur finale) :

- Une conduite raccordée au circuit primaire d’un REP, avec un petit débit à la densité $\rho_{2}$ vers le circuit primaire (dont la densité est $\rho_{1}$ ).

Pour illustrer de façon très simple le type de phénomène qui peut apparaître, prenons le cas d'une conduite horizontale, de diamètre $D$, et prenons comme condition initiale une plaque verticale séparant deux fluides de densité $\rho_{1}$ et $\rho_{2}$. Lorsqu'on retire la plaque, un courant de densité se produit (Fig. 5a) dont on peut calculer approximativement la vitesse $u$ par une théorie potentielle :

$$
u=0,5 \sqrt{2 g \frac{\rho_{1}-\rho_{2}}{\rho_{1}+\rho_{2}} D} .
$$

L'ordre de grandeur de ce résultat est conforme aux résultats de l'expérience de Simpson et Britter [13]. Si l'écoulement dans la conduite a une vitesse $V$ uniforme, il est clair que le courant de densité peut se propager en amont si $V<u$, c'est-à-dire si le nombre de Froude densimétrique $\mathrm{Fr}$ défini par:
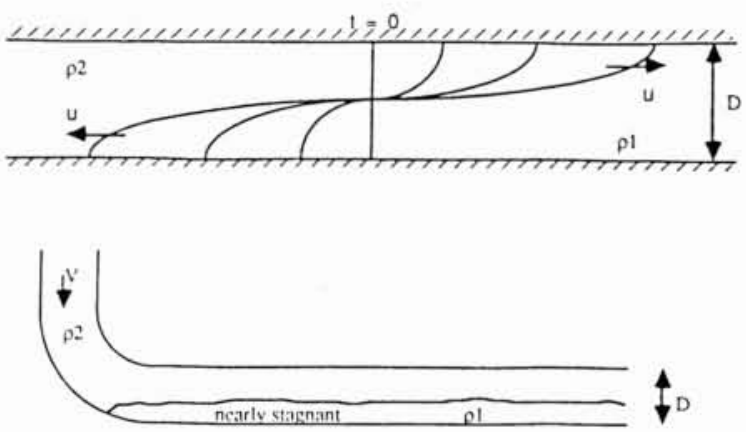

5. (a, haut) Schéma d'un courant de densité dans une conduite. (b, bas) Schéma d'une stratification se terminant dans un coude de la conduite $(\mathrm{Fr}<0,2)$.

$$
\mathrm{Fr}=V \sqrt{2 g \frac{\rho_{1}-\rho_{2}}{\rho_{1}+\rho_{2}} D}
$$

est inférieur à 0,5 . Dans ce cas, le courant de densité peut se propager très loin si la conduite est horizontale, ce qui est de nature à créer une stratification thermique (Fig. 5b). Des expériences simples, par exemple portant sur des écoulements à deux couches ( $\$ 3.2$ et Fig. 8$)$, montrent que quand ce nombre de Froude réduit est petit, il ne peut y avoir de mélange turbulent à l'interface, ce qui assure la stabilité de ces systèmes stratifiés dans les conduites. Les méthodes qui ont été mises au point pour étudier les phénomènes de mélange et de stratification dans des canalisations utilisent les codes tridimensionnels ESTET à EDF (avec une grille curviligne orthogonale de façon à suivre les courbes) et TRIO au CEA.

\subsubsection{Validation pour une expérience de régime transitoire en eau}

La figure 6 montre la validation d'ESTET pour le calcul de phénomènes de stratifications transitoires (Gabillard et Viollet [14]) : cet exemple porte sur l'écoulement suivant 

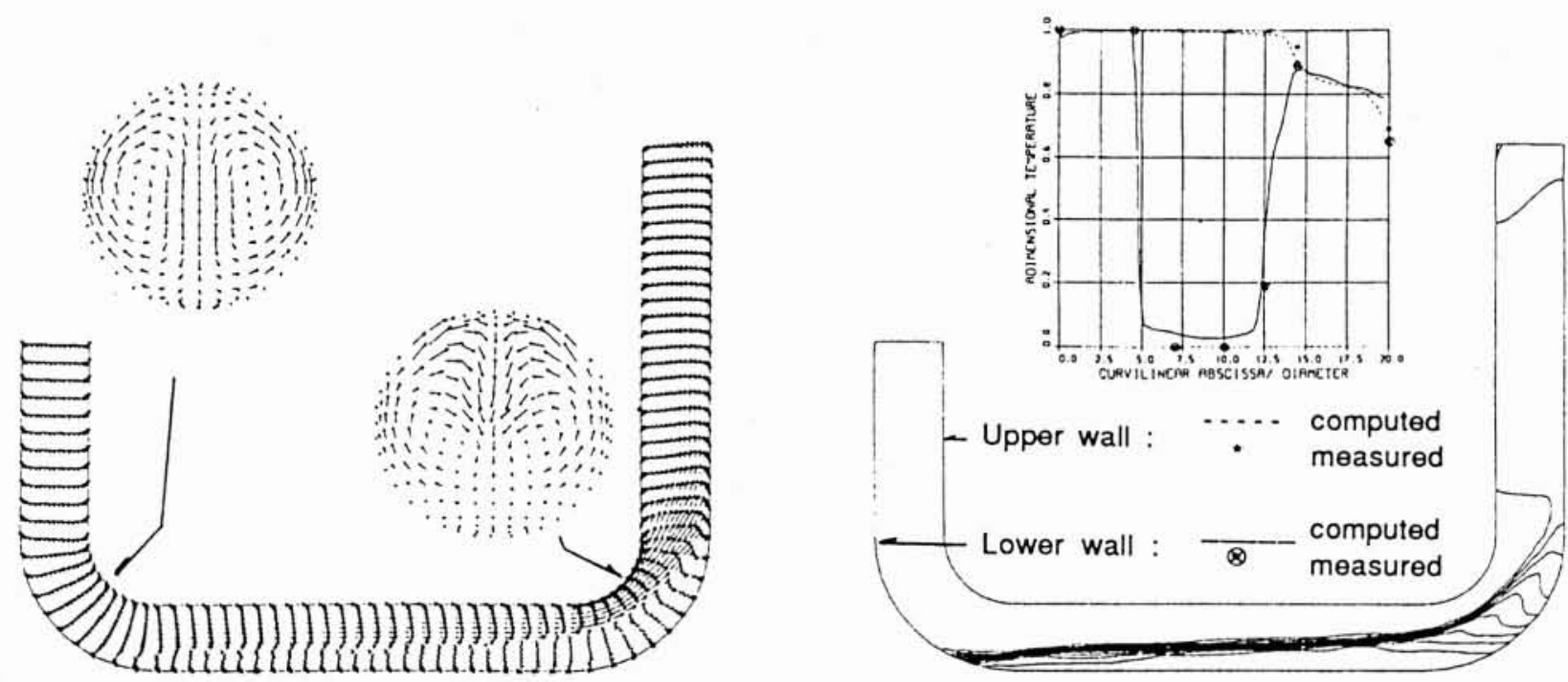

6. (a) Calcul (code ESTET) de l'écoulement $150 \mathrm{~s}$ après une augmentation de la température en amont $(\mathrm{Fr}=0,22)$ : (gauche) vitesse et température calculées ; (droite) température à la paroi calculée et mesurée (source : Gabillard et Viollet [14]).
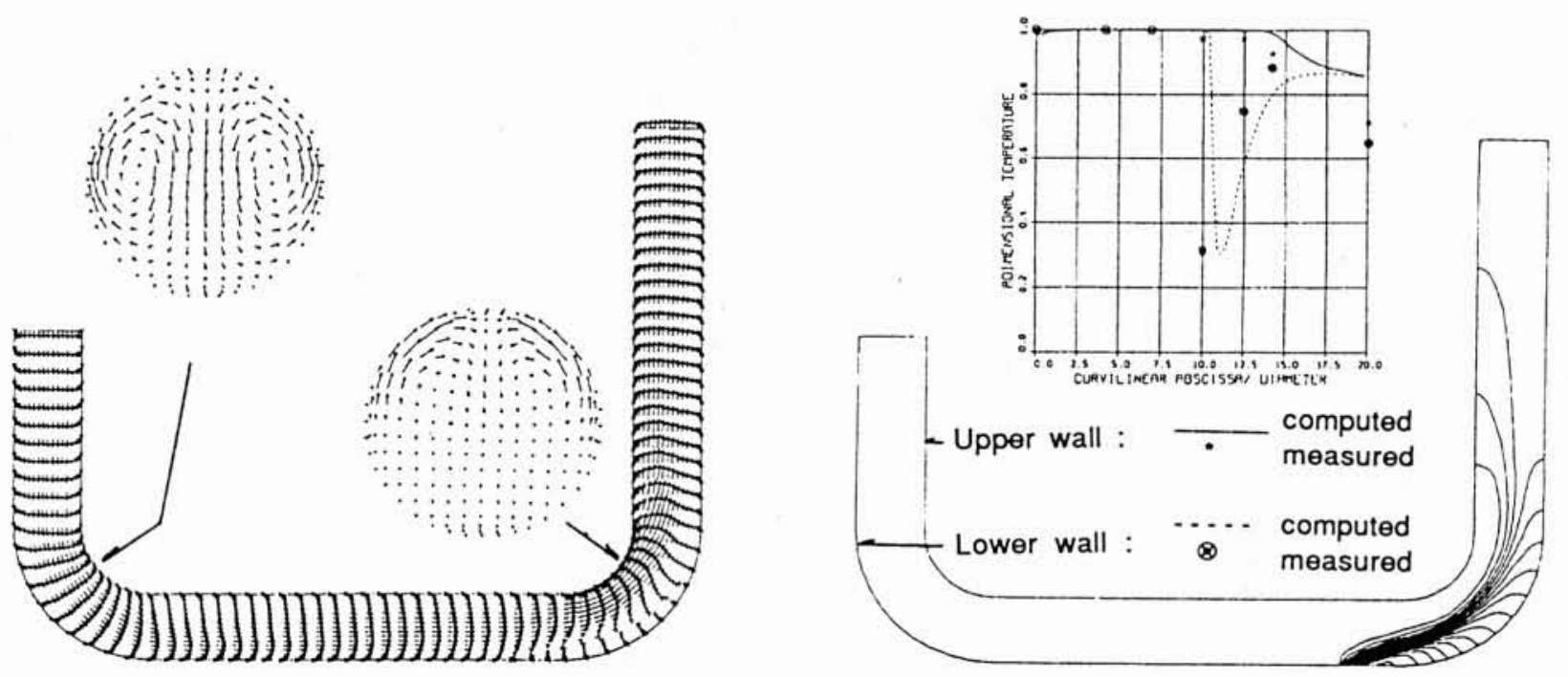

6. (b) Calcul (code ESTET) de l'écoulement $90 \mathrm{~s}$ après une augmentation de la température en amont $(\mathrm{Fr}=0,67)$ : (gauche) vitesse et température calculées ; (droite) température à la paroi calculée et mesurée (source : Gabillard et Viollet [14]).

une augmentation de la température en amont d'une conduite en $\mathrm{U}$, avec deux valeurs différentes du nombre de Froude densimétrique : $\mathrm{Fr}=0,22$ et 0,67 (Fr est défini au $\S 2.5 .1$ ci-dessus). Dans cette expérience (Viollet [15]), le diamètre de la conduite est de $0,25 \mathrm{~m}$.

L'écoulement stratifié et les courants de densité observés sont bien reproduits dans les calculs, par rapport à l'expérience. La modélisation en trois dimensions a permis une amélioration des résultats par rapport aux précédents calculs en deux dimensions (Viollet [15]), surtout pour $\mathrm{Fr}=$ 0,67 , où les courants secondaires tridimensionnels sont plus importants.
2.5.3 Validation pour une expérience de régime transitoire en sodium

Une autre validation de la capacité des modèles de turbulence à fournir une prédiction de la stratification thermique dans des conduites a été effectuée par la comparaison avec les expériences menées sur le sodium NAJET [16] et plus récemment JANUS [17]. Dans ces deux expériences, la stratification est déclenchée par un régime thermique transitoire sur l'écoulement d'entrée. La principale différence entre les deux expériences est la 

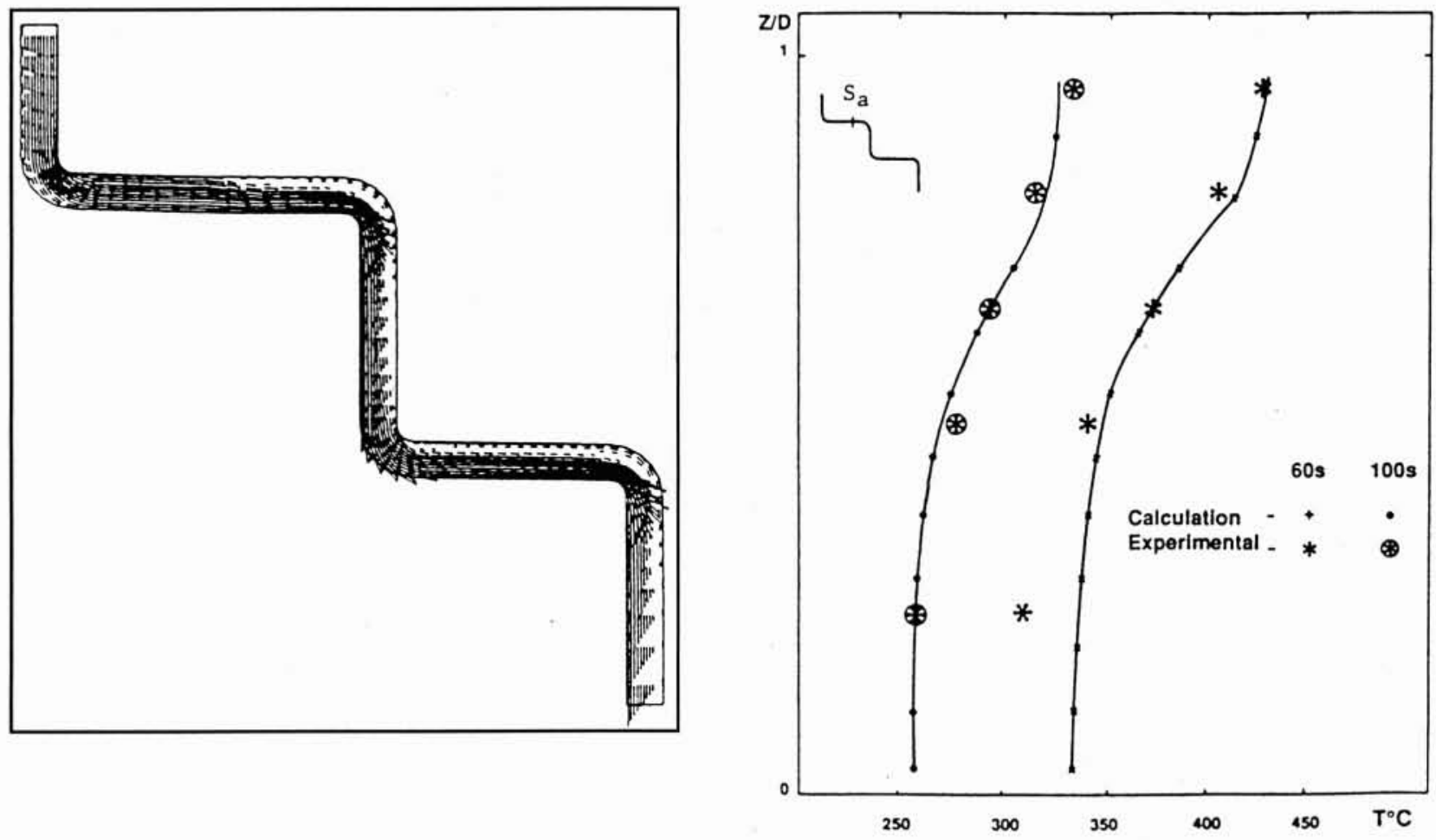

7. Champ de vitesse calculé $100 \mathrm{~s}$ après l'arrivée d'un choc froid et profils de température dans l'expérience JANUS.

géométrie : un U inversé dans le premier cas, un profil en escalier dans le second.

Au cours d'un choc froid, lorsque la température d'entrée diminue, une stratification apparaît si le fluide initialement chaud ne se mélange pas avec le fluide entrant plus froid, et s'élève par convection et par effet de flottabilité dans la partie supérieure de la section horizontale. Dans la géométrie en $U$ inversée, la stratification est plus nette puisque le fluide chaud reste piégé. Dans la géométrie en escalier, le fluide chaud peut s'échapper par la colonne verticale supérieure, ce qui réduit la durée de la stratification.

Dans les deux cas, des simulations numériques avẹc TRIO (ou la version précédente 2D REYCUR) ont montré que le modèle de turbulence permet une bonne prédiction de l'intensité et de la durée de la stratification thermique. C'est ce qu'illustre la figure 7 pour le profil en escalier. Le champ de vitesse $100 \mathrm{~s}$ après l'application du choc froid révèle l'existence d'une zone de recirculation à petite vitesse dans les parties horizontales. La comparaison entre calcul et expérience est présentée sur le profil de température le long d'un diamètre vertical $S_{\mathrm{a}}$.

\section{NOUVEAUX DÉVELOPPEMENTS CON- CERNANT LES MODÈLES DE TURBU- LENCE}

\subsection{Motivation de ces développements}

Le modèle $k-\varepsilon$, basé sur une viscosité turbulente et une diffusivité turbulente a été appliqué, au chapitre précédent, à un certain nombre d'écoulements, généralement avec succès, pour prédire la température moyenne et la vitesse. Toutefois, il faut tenir compte du fait que les hypothèses qui sont liées à ce modèle sont très restrictives dans toute une série de situations, notamment les écoulements en rotation, ou les écoulements stratifiés de façon instable. Schumann [18] a mis en évidence des flux de chaleur à contre-gradient dans des écoulements stratifiés de façon stable (également identifés dans les simulations des grands tourbillons de Khoudli [6]), qui vont au-delà des limites du modèle $k-\varepsilon$ supposant une diffusivité turbulente, mais qui peuvent être importants pour une modélisation fine des écoulements stratifiés. Par ailleurs, la prédiction des fluctuations de température présente une grande importance pratique dans l'analyse des réacteurs ; dans ce contexte, il est important d'élaborer des méthodes de prédiction pour les corrélations turbulentes du second ordre.

D'après Launder, Reece et Rodi [19], on peut écrire le système d'équations de base que l'on peut utiliser pour la prédiction des corrélations du second ordre telles que les tensions de Reynolds $\left(R_{i j}\right)$, les flux turbulents $\left(R_{i f}\right)$, et la variance de température $\left(\overline{T^{\prime} 2}\right)$ de la manière suivante :

$$
\begin{aligned}
\frac{\partial R_{i j}}{\partial t}+\bar{U}_{k} & \frac{\partial R_{i j}}{\partial x_{k}}=-R_{j k} \frac{\partial \bar{U}_{i}}{\partial x_{k}}-R_{i k} \frac{\partial \bar{U}_{j}}{\partial x_{k}}-C_{1} \frac{\varepsilon}{k}\left(R_{i j}-\frac{1}{3} R_{k k}\right) \\
& -C_{2}\left(P_{i j}-\frac{1}{3} P_{k k}\right)-C_{3}\left(G_{i j}-\frac{1}{3} G_{k k}\right) \\
+ & C_{3} \frac{\partial}{\partial x_{k}}\left(\frac{k}{\varepsilon} R_{k l} \frac{\partial R_{i j}}{\partial x_{1}}\right)-\frac{2}{3} \varepsilon \delta_{i j}-\beta g_{\xi} R_{i t} \delta_{j \varepsilon}-\beta g_{\xi} R_{j t} \delta_{i \xi} ;
\end{aligned}
$$




$$
\begin{gathered}
\frac{\partial R_{i t}}{\partial t}+\bar{U}_{k} \frac{\partial R_{i t}}{\partial x_{k}}=-R_{i k} \frac{\partial \bar{T}}{\partial x_{k}}-R_{k t} \frac{\partial \bar{U}_{i}}{\partial x_{k}}-C_{1 t} \frac{\varepsilon}{k} R_{i t}+C_{2 t} R_{k t} \frac{\partial \bar{U}_{i}}{\partial x_{k}} \\
+C_{3 t} G_{i t}+C_{s t} \frac{\partial}{\partial x_{k}} \frac{k}{\varepsilon}\left(R_{l k} \frac{\partial R_{i t}}{\partial x_{1}}+R_{l i} \frac{\partial R_{k t}}{\partial x_{i}}\right)-\beta g_{\xi} \overline{T^{2}} \delta_{i \xi} \\
\frac{\partial \overline{T^{2}}}{\partial t}+\bar{U}_{k} \frac{\partial \overline{T^{2}}}{\partial x_{k}}=-2 R_{k t} \frac{\partial \bar{T}}{\partial x_{k}}+C_{t} \frac{\partial}{\partial x_{k}} \frac{k}{\varepsilon}\left(R_{l k} \frac{\partial T^{2}}{\partial x_{1}}\right)-\varepsilon_{0} .
\end{gathered}
$$

Dans ces expressions, c'est la modélisation la plus simple des corrélations pression-déformation et pression-flux de chaleur qui est utilisée (c'est ce qu'on appelle le modèle IOP pour $\phi_{i j 2}$, les fonctions d'amortissement des fluctuations de pression à la paroi n'étant pas prises en compte). Ces équations (11) ont besoin d'être complétées par des équations relatives aux taux de dissipation $\varepsilon$ et $\varepsilon_{\theta}$. D'après Launder et coll. [19], l'équation du taux de dissipation peut s'écrire :

$$
\begin{aligned}
\frac{\partial \varepsilon}{\partial t}+\bar{U}_{k} \frac{\partial \varepsilon}{\partial x_{k}}=C_{\varepsilon 1} \frac{\varepsilon}{k}\left(\frac{P_{k k}}{2}\right. & \left.+\left(1-C_{\varepsilon 3}\right) \frac{G_{k k}}{2}\right) \\
& +C_{\varepsilon} \frac{\partial}{\partial x_{k}}\left(\frac{k}{\varepsilon} R_{k l} \frac{\partial \varepsilon}{\partial x_{1}}\right)-C_{\varepsilon 2} \frac{\varepsilon^{2}}{k},
\end{aligned}
$$

ou sous la forme plus simple (10) utilisant la viscosité turbulente pour la modélisation du terme de diffusion, en notant que :

$$
\tilde{P}=\frac{P_{k k}}{2} \quad \text { et } \quad \tilde{G}=\frac{G_{k k}}{2} \text {. }
$$

On peut écrire une équation similaire pour le taux de dissipation des fluctuations de température :

$$
\begin{aligned}
\frac{\partial \varepsilon_{\theta}}{\partial t}+\bar{u}_{j} & \frac{\partial \varepsilon_{\theta}}{\partial x_{j}}=\frac{\partial}{\partial x_{j}}\left\{\left(\frac{\kappa t}{\sigma_{\varepsilon \theta}}\right) \frac{\partial \varepsilon_{\theta}}{\partial x_{j}}\right\}+C_{\varepsilon \theta 1} \frac{\varepsilon_{\theta}}{T^{2}} P_{\theta} \\
& -C_{\varepsilon \theta 2} \frac{\varepsilon_{\theta}}{T^{2}} \varepsilon_{\theta}-C_{\varepsilon \theta 3} \frac{\varepsilon}{k} \varepsilon_{\theta}+C_{\varepsilon \theta 4}(\tilde{P}+\tilde{G}) \frac{\varepsilon_{\theta}}{k} .
\end{aligned}
$$

L'équation (13) a été initialement proposée par Launder [20] sans le dernier terme, qui a été suggéré par Chung et Sung [21].
Dans ce qui suit, les résultats sont donnés avec certaines des équations sur les moments du second ordre donnés ci-dessus, par comparaison avec des expériences d'écoulements non isothermes.

\subsection{Modélisation d'un écoulement en cisaillement stra- tifié de façon stable et de façon instable à l'aide d'un modèle au second ordre}

\subsubsection{Définition du cas étudié}

Des écoulements stratifiés horizontaux se produisent dans les grandes chambres de tranquillisation des réacteurs à neutrons rapides refroidis au métal liquide ainsi que dans des phénomènes de mélange à faible débit dans les conduites.

Ce chapitre décrit l'application d'un modèle aux tensions de Reynolds à un écoulement d'eau en canal stratifié de façon stable ou instable. Les résultats expérimentaux ont été obtenus par Viollet [5]. Le canal a une largeur de $0,4 \mathrm{~m}$, et l'épaisseur de chaque couche est $h=0,1 \mathrm{~m}$. Les effets de densité sont obtenus à l'aide d'eau chaude et d'eau froide. Dans les cas stratifiés de façon stable (eau chaude injectée par le haut), $u_{1}=2 u_{2}$, alors que dans les cas stratifiés de façon instable (eau chaude injectée par le bas), $u_{2}=2 u_{1}$ (de la sorte, dans tous les cas, la vitesse de la couche froide est double de la vitesse de la couche chaude). La figure 8 illustre la géométrie de l'expérience. Le paramètre utilisé pour évaluer l'importance des effets de densité est le nombre de Froude (densimétrique) réduit défini comme étant :

$$
F=\left|u_{2}-u_{1}\right| / \sqrt{g\left|\frac{\rho_{2}-\rho_{1}}{\rho_{1}}\right| h .}
$$

3.2.2 Description du modèle de turbulence et de la méthode de résolution numérique

Cette expérience a été calculée par Kanniche et coll. [22]. Les résultats de ces calculs sont résumés ci-dessous.

Le modèle de turbulence utilisé se compose des équations (11) et (12), avec une modélisation simplifiée du taux de dissipation $\varepsilon_{\theta}$, selon Launder [20], en supposant un ratio constant $R$ entre les échelles de longueur turbulente dynamique et thermique. Cela permet une expression algébrique simple pour $\varepsilon_{\theta}$ :

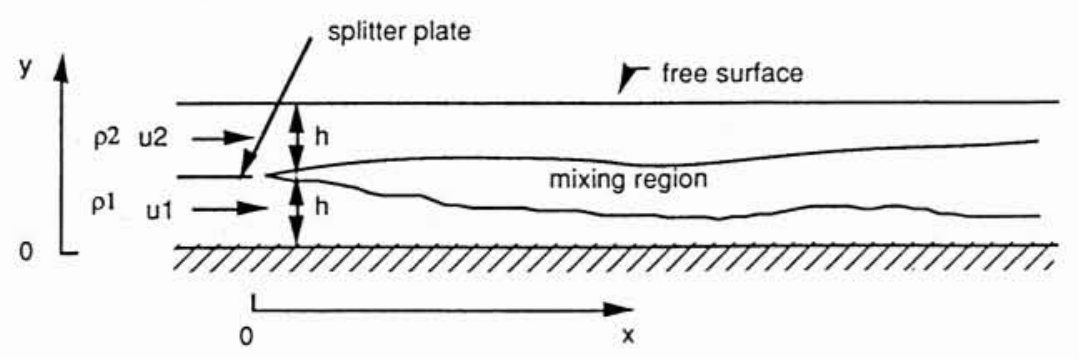

8. Schéma définissant l'expérience d'écoulement stratifié de Viollet [5]. 


$$
\varepsilon_{\theta}=\frac{1}{R} \varepsilon \overline{\frac{T^{\prime 2}}{k}} .
$$

où $R \sim 0,5$ à 0,8 (d'après les données relatives à l'amortissement de la turbulence dans la grille chauffée).

L'ensemble des constantes utilisées est donné cidessous, et provient des travaux de précédents chercheurs (principalement Hossain et Rodi [23]) :

\begin{tabular}{|l|c|c|c|c|c|c|}
\hline$C_{l}$ & $C_{2}$ & $C_{3}$ & $C_{s}$ & $C_{l t}$ & $C_{2 t}$ & $C_{3 t}$ \\
\hline 2,2 & 0,55 & 0,55 & 0,25 & 3,2 & 0,5 & 0,5 \\
\hline$C_{s t}$ & $C_{t \prime}$ & $C_{r 2}$ & $C_{\varepsilon}$ & $C$ & $R$ & \\
\hline 0,11 & 1,44 & 1,92 & 0,15 & 0,11 & 0,5 & \\
\hline
\end{tabular}

Jusqu’à présent, la stabilisation du couplage entre toutes les équations concernées, dans des méthodes de résolution avec une diffusion numérique faible, présentait des difficultés numériques très importantes (il ne faut pas oublier que la diffusion numérique peut stabiliser la méthode numérique mais annule l'intérêt d'une modélisation de la turbulence). Des méthodes de résolution stables apparaissent à présent (Huang et Lechziner [24]: Kanniche et coll. [25]). La méthode de résolution est comparable à celle qui est utilisée dans le code ESTET (Cf. Annexe), mais tous les termes sources des onze équations couplées sont traités de manière couplée en faisant appel à une méthode de Gauss-Seidel pour la résolution du système linéarisé. Dans cette expérience, les choix numériques pour le traitement couplé de toutes les équations de transport en ce qui concerne la vitesse moyenne, la température moyenne, les tensions de Reynolds, les flux turbulents, la variance des fluctuations de température, le taux de dissipation ont été jugés suffisamment robustes pour le traitement de cet écoulement complexe.

\subsubsection{Résultats}

La grille utilisée pour ce calcul comporte 125 points sur l'axe des $x$ et 30 sur l'axe des $y$ et va jusqu'à $x / h=30$. Les figures $9 \mathrm{a}$ et $9 \mathrm{~b}$ montrent les profils verticaux mesurés et calculés de la température moyenne dans des écoulements stratifiés de façon stable et de façon instable avec des effets de flottabilité faibles $(F=5)$ et forts $(F=0,9)$.

La figure $9 \mathrm{c}$ montre, d'après le calcul, l'anisotropie de la turbulence et l'énergie cinétique turbulente, prises à mi-hauteur du canal $(y / h=1)$. Dans l'écoulement stratifié de façon stable $(F=0,9)$, les effets de flottabilité empêchent complètement le mélange turbulent, situation à laquelle aboutissaient aussi les calculs précédents utilisant le modèle $k-\varepsilon$ et un modèle algébrique aux tensions de Reynolds [5]. Le résultat calculé montre que l'on peut détecter un flux de chaleur à contre-gradient dans la partie supérieure de l'écoulement: ce type de résultat a déjà été analysé par Schumann [18] comme découlant du fait que le taux de dissipation des fluctuations de température est trop faible pour compenser les autres termes de l'équation de la variance de la température fluctuante. Là où le concept de viscosité turbulente a un sens, le nombre de Richardson du flux reste de l'ordre de 0,25 , et le nombre de Prandtl turbulent augmente en même temps que $x$. Dans l'écoulement stratifié de façon instable $(F=0,9)$, on observe un mélange très fort, suivi d'une inversion du gradient de température (effet typique de flux de chaleur à contre-gradient). Le très fort mélange était mal représenté par les calculs précédents sur la base du modèle $k-\varepsilon$ et se trouve mieux représenté lorsqu'on utilise le modèle algébrique aux tensions de Reynolds [5]. Bien entendu, ni l'un ni l'autre de ces modèles ne permet de reproduire le passage d'un écoulement instable à un écoulement stable. Les présents résultats, sur la base d'un modèle de transport des tensions de Reynolds, montrent qu'on obtient bien l'ensemble du schéma de l'écoulement, fort mélange suivi d'une inversion de température (passage d'un écoulement stratifié de façon instable à un écoulement stratifié stable), mais que le taux de mélange reste légèrement sous-évalué par les prédictions dans la zone de fort mélange. Dans la zone stratifiée de façon instable $(x / h<15)$, le nombre de Prandtl turbulent calculé tombe à environ 0,5 et la valeur maximum du nombre de Richardson du flux ${ }^{3}$ est de l'ordre de -2,8. La composante verticale des fluctuations de vitesse augmente par les effets de flottabilité dans la zone de début de l'écoulement (plus loin, on observe une redistribution vers les fluctuations longitudinales), et il peut être intéressant de remarquer que l'anisotropie se trouve bien corrélée avec le nombre de Richardson du flux.

\subsection{Modélisation des flux de chaleur turbulents et des fluctuations de température}

Dans une thèse récente faite au CEA en collaboration avec l'Ecole Centrale de Lyon, Mompean [26] proposait une autre approche de la résolution du système complet d'équations donné au $\$ 3.1$

Dans cette approche, on résoud le système complet d'équations pour les quantités turbulentes concernant le champ de température, c'est-à-dire les équations de transport pour les flux thermiques turbulents $R_{i p}$, les fluctuations de température $\overline{T^{2}}$ et le taux de dissipation des fluctuations de température $\varepsilon_{0}$. La partie hydraulique du champ turbulent est résolue uniquement à l'aide du modèle $k-\varepsilon$ et non à l'aide des équations de transport pour les tensions turbulentes. En conséquence, tous les termes relatifs à la diffusion dans les équations de transport pour les moments du second ordre $R_{i r}, \overline{T^{2}}$ et $\varepsilon$ sont modélisés à partir d'une diffusivité scalaire turbulente. On supprime ainsi une des causes des difficultés numériques rencontrées par les modèles numériques, sans trop perdre en précision puisque ces termes de diffusion turbulente sont généralement dominés par la production et la dissipation voire par la corrélation avec les fluctuations de pression. Cette approximation peut exercer une plus grande influence sur les contributions des tensions de Reynolds aux termes relatifs à la production.

Le principal avantage de ce modèle tient à la résolution d'une équation de transport pour la dissipation des fluctuations de température $\varepsilon_{\theta}$. Or, on attend de la résolution d'une équation de transport pour $\varepsilon_{\theta}$ qu'elle permette d'améliorer la prédiction des fluctuations de température. Sans cette équation de transport, $\varepsilon_{0}$ doit être déduit de la

$\left(^{3}\right)$ Le nombre de Richardson du flux est un paramètre local défini comme : $R_{f}=-\tilde{G} / \tilde{P}$. Il ne faut pas le confondre avec le nombre de Richardson (global) Ri défini à partir du nombre de Froude densimétrique $\mathrm{Fr}$ comme étant : $\mathrm{Ri}=-1 / \mathrm{Fr}^{2}$. 

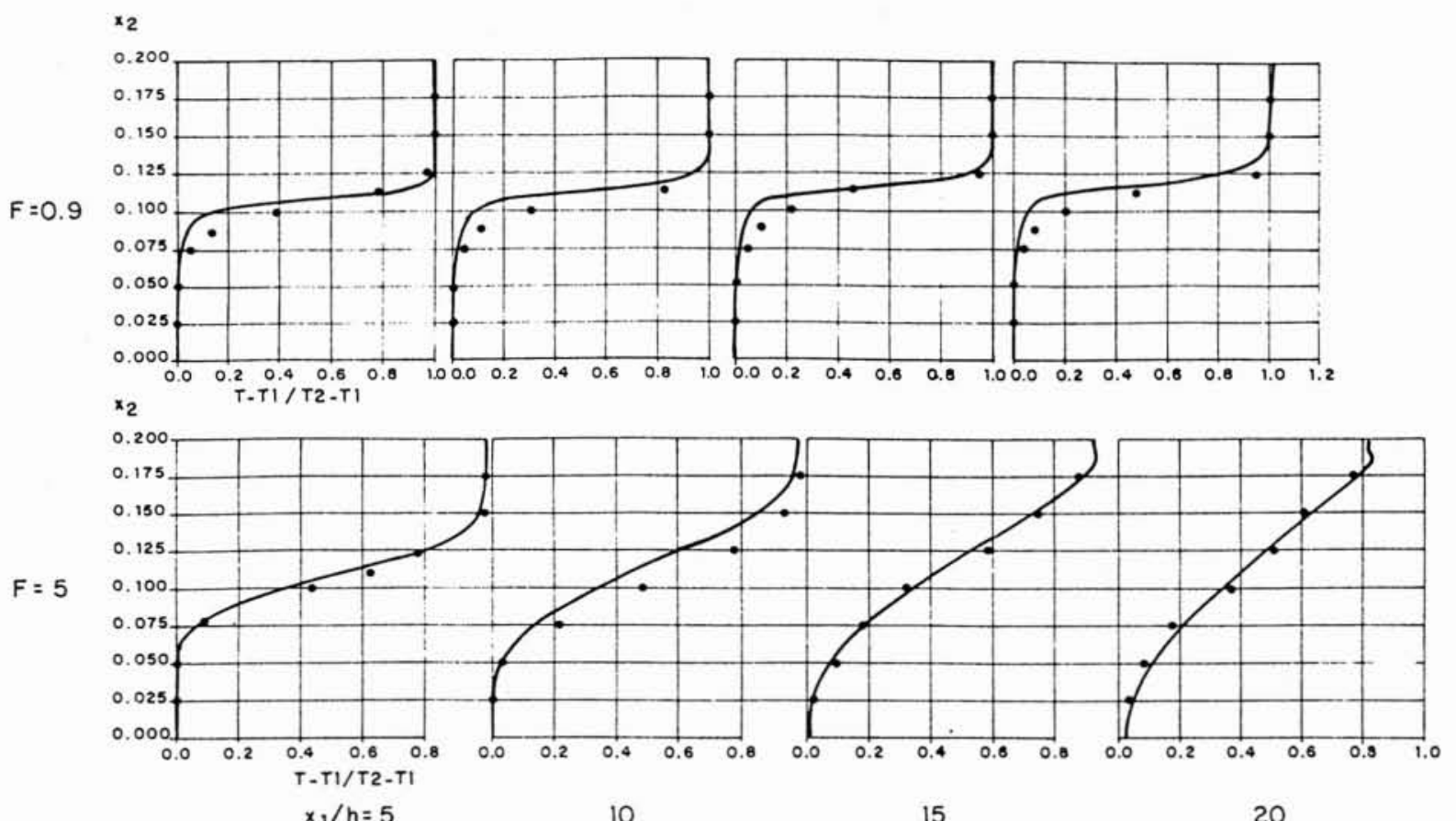

$x_{1} / h=5$

10

15

20
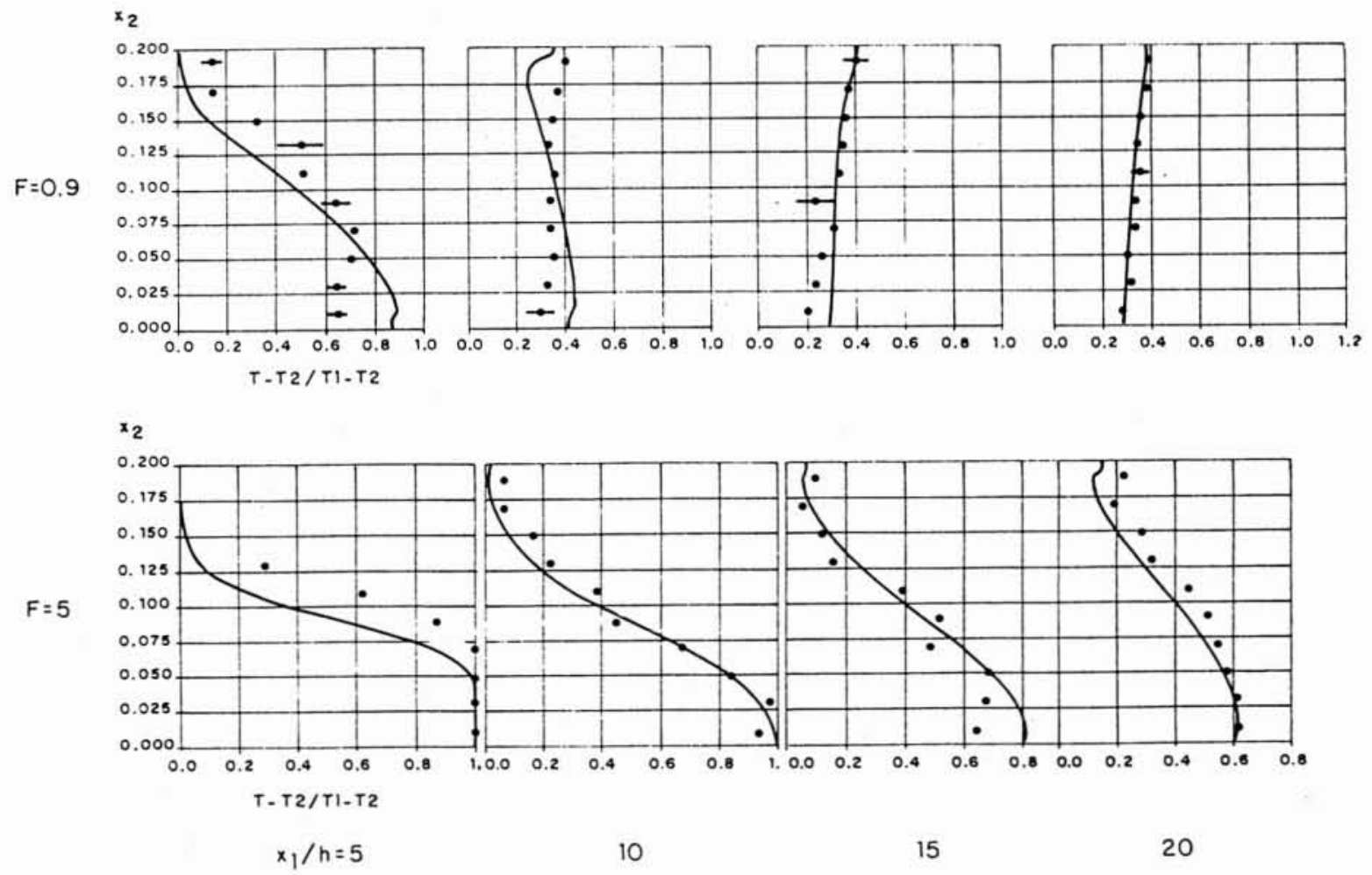

9. (a, haut) écoulement stratifié stable, $F=0,9$ et $F=5$. Profil vertical calculé (traits pleins) et mesuré de la température moyenne à $x / h=5,10,15,20$ (de gauche à droite) (source : Kanniche et coll. [22]).

(b, bas) Ecoulement stratifié instable, $F=0,9$ et $F=5$. Profil vertical calculé (traits pleins) et mesuré de la température moyenne à $x / h=5,10,15,20$ (de gauche à droite) (source : Kanniche et coll. [22]). 

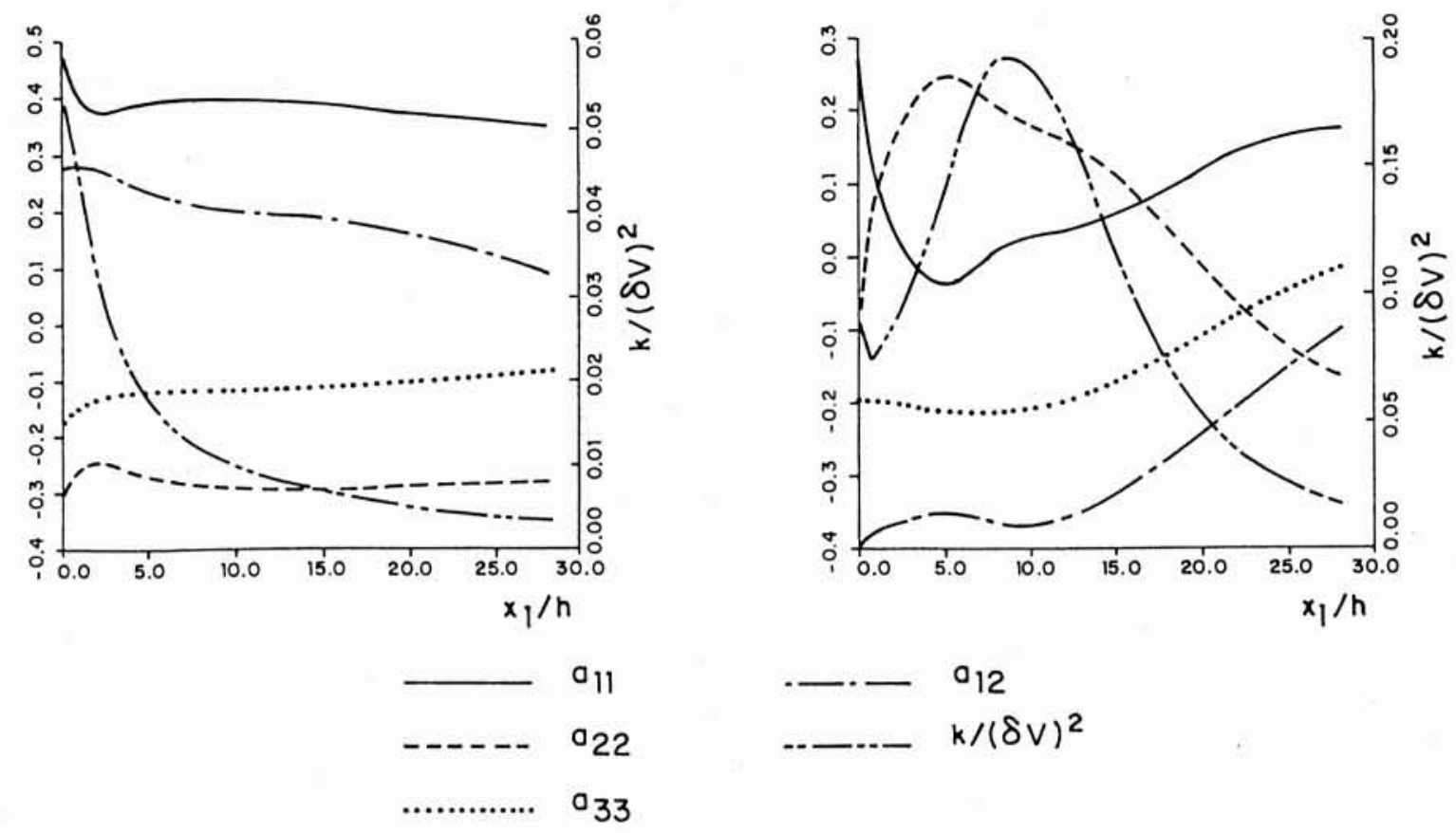

9. (c) Représentations graphiques de l'énergie cinétique turbulente adimensionnelle : $k /\left(u_{2}-u_{1}\right)^{2}$ et de l'anisotropie de la turbulence : $a_{i j}=R_{i j} / k-2 \delta_{i j} / 3$ en fonction de $x$, à $y / h=1$, déduites des calculs du second ordre $(F=0,9)$. A gauche : cas stable; à droite : cas instable (source : Kanniche et coll. [22]).
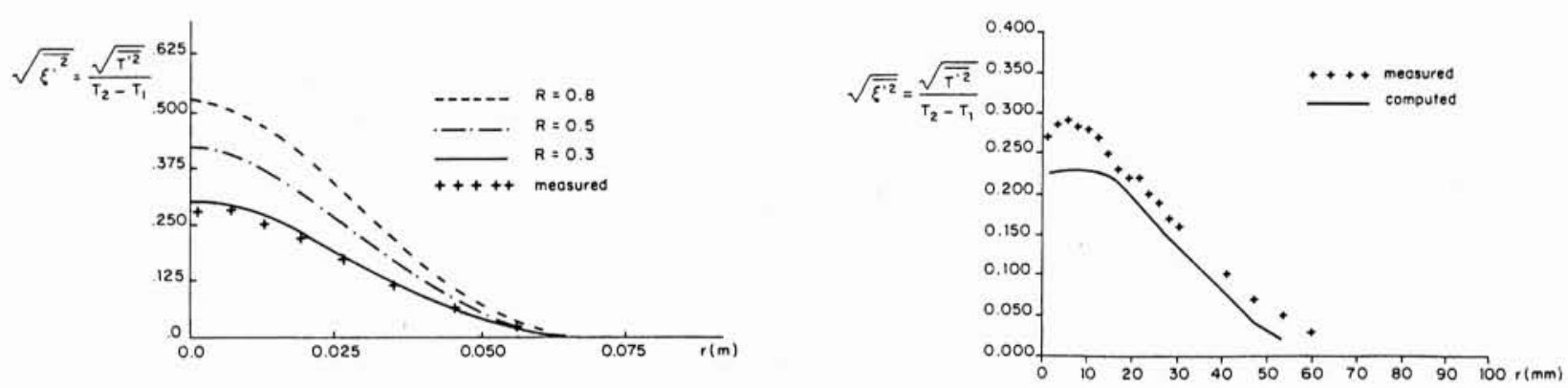

10. (a) Influence de $R$ sur le profil de variance des fluctuations de température calculées à l'aide de l'équation (14). Jet axisymétrique assez chaud, $Z / D=0,8$ (source : Mompean [26]). (b) Comparaison des mesures et des calculs (à l'aide de l'équation (13)) pour la variance des fluctuations de température. Jet axisymétrique assez chaud, $Z / D=0,8$ (source : Mompean [26]).

relation algébrique (14), où $R$ est un nombre adimensionnel, correspondant au rapport des deux échelles de temps pour l'amortissement des fluctuations de vitesse et de température. Les valeurs de $R$ peuvent varier sensiblement avec le type d'écoulement considéré, et une équation de transport de $\varepsilon_{\theta}$ est censée être plus précise pour suivre ces variations.

Le modèle a été validé pour des situations d'écoulements élémentaires :

- amortissement d'une turbulence de grille,

- mélange d'un jet chauffé dans un fluide ambiant stagnant (comparé à une expérience en air),

- mélange de deux jets coaxiaux avec des températures inégales et des vitesse égales (comparé à une expérience en sodium).

Mompean [26] rapporte les résultats de ces validations. Ils montrent, dans la plupart des situations d'écoulement considérées, une amélioration de la prédiction des fluc- tuations de température et des écoulements turbulents avec une équation de transport pour $\varepsilon_{\theta}$.

Les résultats concernant le mélange d'un jet turbulent dans un fluide ambiant stagnant sont présentés ci-après. Les résultats expérimentaux ont été obtenus à l'Institut de Mécanique Statistique de la Turbulence de Marseille. Le montage expérimental se compose d'un jet d'air circulaire chauffé ; la température et les vitesses ont été mesurées sur des lignes transversales radiales à différentes positions axiales.

Les profils du flux de chaleur radial et de la variance de température sont comparés à une position axiale située à huit diamètres de la sortie du jet. Les quantités turbulentes sont rendues adimensionnelles avec la vitesse débitante calculée dans la section initiale du jet et la différence de température maximum $\delta T=T_{2}-T_{1}$.

A des fins de comparaison, la figure 10a montre la variance des fluctuations de température prédites par un 
modèle utilisant une valeur donnée de $R$ (avec l'équation (4) au lieu de l'équation (13)). On trouve la meilleure correspondance pour une valeur de $R=0,3$, ce qui est très différent de la valeur $R=0,8$ déduite de l'amortissement d'une turbulence de grille.

Au contraire (Cf. Fig. 10b), le modèle utilisant l'équation de transport (13) pour $\varepsilon_{\theta}$ permet de prédire les deux situations avec la même série de constantes empiriques:

\begin{tabular}{|l|c|c|c|}
\hline$C_{t \theta 1}$ & $C_{t \theta 2}$ & $C_{\varepsilon \theta 3}$ & $C_{\varepsilon \theta 4}$ \\
\hline 1,96 & 2 & 0,8 & 0,8 \\
\hline
\end{tabular}

EDF (Peniguel et Lacroix [27]) est parvenu aux mêmes conclusions, qui montrent tout l'intérêt d'un modèle utilisant des équations de transport pour $k, \varepsilon, \overline{T^{2}}$ et $\varepsilon_{\theta}$, dans le cas d'une couche stratifiée stable, comme le montre la figure 8 (avec comme constantes: $C_{\varepsilon \theta 1}=1,48$; $\left.C_{\varepsilon \theta 2}=1,48 ; C_{\varepsilon \theta 3}=0,35 ; C_{\varepsilon \theta 4}=0\right)$. La figure 11 illustre l'évolution, en aval, de la variance des fluctuations de température.

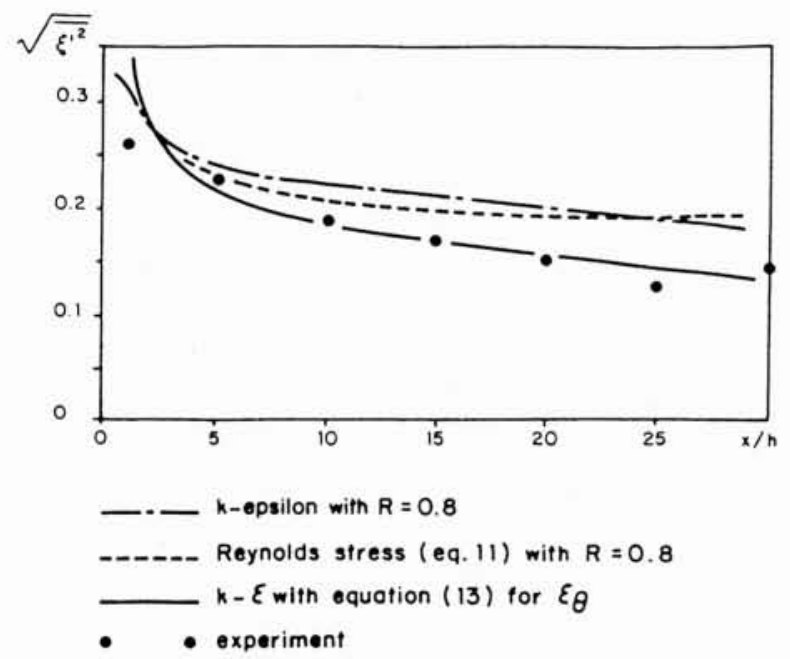

11. Représentations graphiques de $\sqrt{\zeta^{\prime 2}}=\sqrt{\overline{T^{2}}} /\left(T_{1}-T_{2}\right)$ calculé et mesuré sur la ligne $y / h=1$ dans l'écoulement stratifié en canal illustré à la fig. 8, avec une stratification stable et $\boldsymbol{F}=\mathbf{5}$ (effet de flottabilité modéré). $L$ 'expérience est comparée à divers modèles pour $R_{i j}, R_{i t}$ et $\varepsilon$.

Les valeurs expérimentales de l'intensité turbulente sont mieux décrites par l'équation de transport sur $\varepsilon_{\theta}$, alors qu'une valeur constante de $R=0,8$ sous-évalue la prédiction de l'amortissement des fluctuations de température.

\subsection{Fluctuations de température dans les métaux liqui- des}

L'application des modèles de turbulence à des métaux liquides exige la prise en compte du caractère particulier de ces fluides dû à leur forte conductivité thermique (ou faible nombre de Prandtl). Le nombre de Péclet turbulent est approximativement inférieur de trois ordres de grandeur au nombre de Reynolds turbulent. Ainsi, dans de nombreuses situations d'écoulement, le nombre de Péclet n'est pas suffisamment élevé pour que l'on puisse tenir pour négligeables les effets moléculaires, même si le nombre de Reynolds est suffisamment élevé pour que l'on puisse tenir pour négligeables les effets visqueux. C'est ce qui distingue les métaux liquides des fluides ordinaires dont le nombre de Prandtl est proche de l'unité. Dans ce dernier cas, les effets moléculaires deviennent négligeables en même temps dans le champ dynamique et dans le champ thermique.

Une première conséquence concerne le profil de température dans les couches turbulentes à la paroi : l'extension de la sous-couche conductive est beaucoup plus grande que la sous-couche visqueuse (Cf. [28]). Le traitement des conditions aux limites dans les codes numériques (fonctions de paroi,...) doit être revu en conséquence.

Une autre conséquence concerne la modélisation des équations de transport des flux turbulents, des fluctuations de température et du taux de dissipation des fluctuations de température présentées au $\$ 2.1$. Comme le signalait Pimont [29], l'hypothèse de l'isotropie des échelles dissipatives généralement utilisée dans les modèles de turbulence peut se trouver invalidée pour des métaux liquides.

La figure 12 compare les échelles de longueur turbulentes pour deux fluides $\left(\mathrm{P}_{\mathrm{r}} \sim 1\right.$ et $\left.\mathrm{P}_{\mathrm{r}} \ll 1\right)$. Pour le petit nombre de Prandtl, les microéchelles thermiques se situent entre la macro-échelle 1 et la micro-échelle $\lambda$ de Taylor. Les micro-échelles thermiques se rapprochent de la macroéchelle lorsque le nombre de Prandtl diminue. Un nombre de Reynolds élevé n'est pas une condition suffisante pour assurer l'isotropie des petites échelles thermiques.

Par une approche systématique des équations de transport pour des écoulements turbulents, Pimont a déduit des termes supplémentaires dus à l'anisotropie des petites échelles des fluctuations de température.

Ces termes supplémentaires sont :

- un terme de dissipation dans l'équation de transport pour les flux turbulents $R_{i t}$ :

$$
\varepsilon_{i t}=M_{1} \sqrt{\frac{\varepsilon \varepsilon_{\theta}}{k}} \sqrt{\frac{k}{T^{\prime 2}}} D_{i j} R_{j t},
$$

où $D_{i j}$ est le taux de déformation du mouvement moyen ;

- deux termes supplémentaires dans le transport pour le taux de dissipation thermique $\varepsilon_{\theta}$. Ces termes représentent respectivement :

(a) l'action du gradient de température moyen sur la turbulence :

$$
P_{T, \varepsilon \theta}=-4 M_{1} \sqrt{\frac{\varepsilon}{k} \frac{\varepsilon_{\theta}}{\overline{T^{\prime 2}}}} \sqrt{\frac{k}{\varepsilon}-D_{i j} R_{j t} \nabla_{i} \bar{T} .}
$$

(b) l'action du taux de déformation moyen sur la turbulence : 


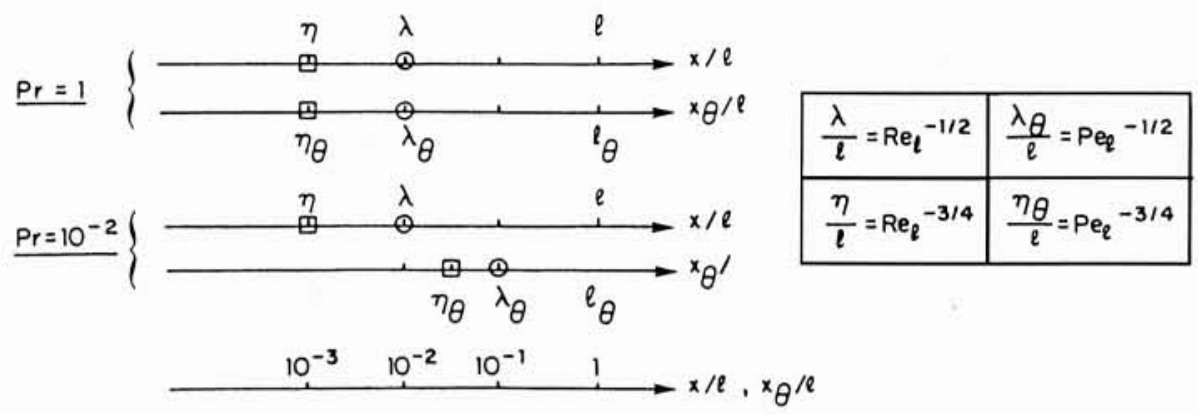

$$
\mathrm{Re}_{l}=10^{4} \quad \mathrm{Pe}_{\ell}=\operatorname{Pr} \mathrm{Re}_{\ell}
$$

12. Comparaison des échelles de longueur turbulentes pour deux fluides $\left(\operatorname{Pr}=1\right.$ et $\left.\mathrm{P}_{\mathrm{r}} \ll 1\right)$. $\lambda$ est la micro-échelle de Taylor, $\eta$ est l'échelle de longueur dissipative de Kolmogorov et $\eta_{\theta}$ l'échelle de longueur dissipative des fluctuations de température.

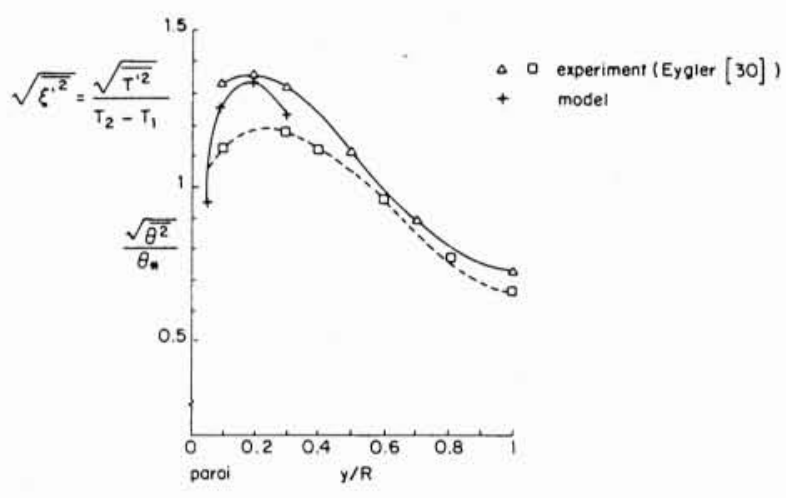

13. Distribution radiale de la variance des fluctuations de température : comparaison entre modèle et expérience (Pimont [29]).

$$
P_{U, \varepsilon \theta}=-M_{2, \varepsilon \theta} \sqrt{\frac{\varepsilon}{k}} D_{i j} D_{j i}
$$

Une solution analytique du modèle complet a été déduite dans le cas d'un écoulement en conduite pleinement développé. La figure 13 montre, pour les fluctuations thermiques, une comparaison de ce modèle avec les mesures effectuées dans du mercure [30].

\subsection{Autres développements sur les fluctuations de tem-} pérature

La prédiction des fluctuations de température peut être très importante pour l'analyse mécanique des structures solides. En effet, ces fluctuations de température peuvent être causées soit par un mélange turbulent, soit par des zones de gravité à l'interface d'une stratification. Seul le premier cas sera évoqué ici. Du point de vue de l'analyse mécanique, deux éléments d'information doivent être utiles : la fonction de densité de probabilité de la température instantanée, et le spectre de fréquence des fluctuations de température.
Aux chapitres précédents, des résultats ont été présentés pour la variance des fluctuations de température. L'objet de ce qui suit est de montrer comment cet élément d'information peut être traité pour obtenir sous une forme approximative des informations plus détaillées nécessaires à l'analyse mécanique.

(a) La fonction de densité de probabilité (pdf) de la température instantanée, $P(T)$. La signification de cette pdf est la suivante: $P(T) \mathrm{d} T$ est la probabilité de trouver une température dans l'intervalle $[T, T=\mathrm{d} T]$. Cette fonction est donc le seul critère objectif permettant de définir l'amplitude des fluctuations de température. Si l'on considère le mélange de deux écoulements avec des températures $T_{1}$ et $T_{2}$ par exemple, $P(T)$ est égal à zéro si $T<T_{1}$ et $T>T_{2}$, mais la probabilité de trouver une température entre $T_{1}$ et $T_{2}$ peut être faible, tout en étant différente de zéro: à cet égard, cela n'a guère de sens de dire que la température instantanée varie entre $T_{3}$ et $T_{4}$ sans définir la probabilité y afférant. Des équipes de recherche travaillant sur la modélisation de la combustion turbulente [16] ont entrepris de modéliser la forme de la pdf par la résolution d'une équation de transport, ce qui pourrait déboucher sur des méthodes applicables au présent domaine d'étude. Une manière plus simple de définir un modèle pour la pdf est de présumer sa forme, puis d'ajuster les paramètres de cette forme présumée à partir de la connaissance de la température moyenne calculée et d'une variance calculée des fluctuations de température. Les courbes de Gauss ne sont pas très pratiques en raison du caractère limité des fluctuations de température (entre $T_{1}$ et $T_{2}$ ); en revanche, le choix de fonctions beta (couramment utilisées dans la modélisation de la combustion) pourrait convenir. Cette pdf de type beta s'écrit :

$$
P(\xi)=\frac{\xi^{\mathrm{a}-1}(1-\xi)^{\mathrm{b}-1}}{\int_{0}^{1} \xi^{\mathrm{a}-1}(1-\xi)^{\mathrm{b}-1} \mathrm{~d} \xi} \text { où }
$$

$\xi=\frac{T-T_{1}}{T_{2}-T_{1}} \quad$ (température adimensionnelle)

et avec: $\quad a=\xi \frac{\xi(1-\xi)-\xi^{\prime 2}}{\xi^{2}}$ 


$$
\mathrm{b}=(1-\xi) \frac{\xi(1-\xi)-\xi^{\prime 2}}{\xi^{\prime 2}}
$$

(b) Le spectre des fluctations de température fournit des informations sur les fréquences rencontrées, avec le niveau d'énergie $E_{f}$ attaché à chaque intervalle de fréquence $(f, f+\mathrm{d} f)$ :

$$
\overline{T^{\prime 2}}=\int_{0}^{\infty} E_{f}(f) \mathrm{d} f
$$

Le seul outil de calcul capable de prédire la forme du spectre de fluctuations de température $E_{f}(f)$ est la simulation directe (pour des écoulements à faible nombre de Reynolds) ou la simulation des grands tourbillons (qui ne prédit que la partie basse fréquence du spectre). Ces méthodes fournissent désormais des résultats présentant un très bon niveau de précision, mais restent d'un maniement lourd, et sont encore en partie limitées aux écoulements très simples. On peut déduire de la théorie de la turbulence isotrope une forme modélisée du spectre de température (Cf. Hinze [31]) qui, malgré certaines inexactitudes liées à l'hypothèse de turbulence isotrope, est utile dans un certain nombre d'applications. Le spectre de fréquence des fluctuations de température $E_{f}(f)$ est lié au spectre tridimensionnel $E(k)$, à condition que la vitesse moyenne $u$ soit supérieure aux fluctuations de la vitesse (de sorte qu'un spectre de fréquence puisse être considéré comme un spectre spatial unidimensionnel) de :

$$
E_{f}(f)=\frac{2 \pi}{u} \int_{2 \pi f / u}^{\infty} \frac{E(k)}{k} \mathrm{~d} k,
$$

où $k=\sqrt{k \vec{k}}$ est le module du nombre d'onde tridimensionnelle $\vec{k}$.

Si les nombres de Reynolds et de Péclet sont l'un et l'autre suffisamment grands (Cf. Fig. 14), le spectre présente une gamme inertielle où :

$$
E(k)=\beta \varepsilon_{\theta} \varepsilon^{-1 / 3} k^{-5 / 3}
$$

( $\varepsilon_{\theta}$ est le taux de dissipation de la variance $\overline{T^{\prime 2}}$, et $\beta \sim 0,5$ ).

Si le nombre de Prandtl est de l'ordre de l'unité (eau, par exemple), cette gamme inertielle est suivie, aux fréquences supérieures, d'une plage dissipative dans laquelle les fluctuations de température et de vitesse font l'objet d'une dissipation visqueuse et diffusive.

Dans les fluides à faible nombre de Prandtl (sodium, par exemple), la gamme inertielle est suivie d'une gamme inertielle «de diffusion ", où les fluctuations de température sont dissipées par conduction thermique mais où les fluctuations de vitesse continuent à échapper à la dissipation visqueuse ; dans cette gamme inertielle « de diffusion ", le spectre peut être noté approximativement :

$$
E(k)=\beta \varepsilon_{\theta} \varepsilon^{-1 / 3} k^{-5 / 3} \exp \left\{-\frac{3}{2} \beta\left(k \eta_{\theta}\right)^{4 / 3}\right\},
$$

avec $\eta_{\theta}$ l'échelle de longueur thermique de Kolmogorov :

$$
\eta_{\theta}=\kappa \frac{3}{4} / \varepsilon^{1 / 4}
$$

D'autres théories font état d'une loi en puissance $k^{17 / 3}$ pour cet intervalle.

\section{IV $\square$ CONCLUSION}

En raison des progrès réalisés ces dernières années dans les techniques numériques, il est désormais possible d'obtenir des méthodes de résolution précises des équations de la dynamique des fluides. Raffiner le maillage est, dans de nombreux cas, le moyen pratique de vérifier la précision de la solution numérique. On peut mettre en œuvre cette technique sur des calculs tridimensionnels grâce à l'accroissement de la vitesse et de la capacité de mémoire des supercalculateurs.
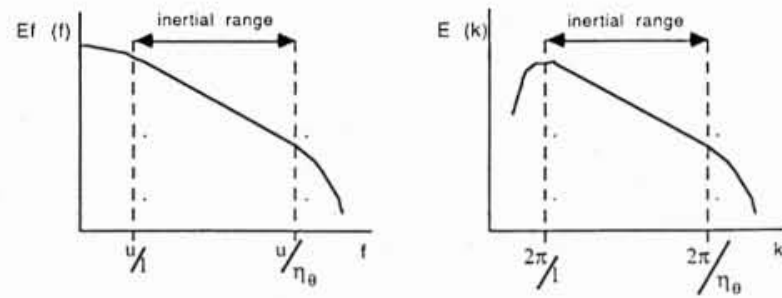

14. Spectre de fréquence et spectre tridimensionnel des fluctuations de température. Dans ce cas de figure, les nombres de Reynolds et de Péclet sont supposés suffisamment élevés. $l$ est l'échelle de longueur des grands tourbillons.

La possibilité d'obtenir des solutions avec une erreur numérique faible permet d'utiliser couramment des calculs numériques tridimensionnels dans le cadre de projets de thermohydraulique. La sûreté d'utilisation de logiciels tels que ESTET, TRIO ou N3S permettant des calculs en tridimensionnel, exige que soient menées à bien des études de validation détaillées sur la base d'abord d'écoulements turbulents dans des géométries simples, puis de situations plus complexes, plus proches de celles qu'on rencontre en pratique dans les analyses de réacteurs.

Un des points particuliers à valider a été le choix du modèle de turbulence et des conditions aux limites à la paroi. Le modèle $k-\varepsilon$ à deux équations est aujourd'hui utilisé dans toutes les applications pratiques, d'une façon relativement sûre grâce aux grands efforts de validation menés par la communauté internationale spécialisée dans la mécanique des fluides numérique, ainsi qu'à EDF et au CEA.

Pour le traitement des cas se situant à la limite ou en dehors du domaine de validité du modèle $k-\varepsilon$ - écoulements avec effets de diffusion à contre-gradient dans des écoulements stratifiés de façon instable, écoulements en rotation ou soumis à de fortes accélérations on a mis au point et intégré aux codes de calcul des méthodes de prédiction utilisant les équations de transport pour les flux de chaleur turbulents et les tensions de Reynolds. Utilisées conjointement avec l'équation de transport pour la variance des fluctuations de température et avec le taux de dissipation des fluctuations de température, ces méthodes permettent de décrire certains paramètres statistiques du champ des fluctuations de température, ce qui est important pour l'analyse des contraintes mécaniques d'origine thermique. 
On peut espérer qu'à l'avenir une prédiction spectrale complète des fluctuations de température sera possible avec l'accroissement de l'utilisation de simulations directes ou de simulations des grandes échelles dans des écoulements industriels complexes.

\section{Annexe 1 - Méthodes numériques utilisées à EDF dans les systèmes ESTET et N3S de simulations d'écoulements 3D}

Pour résoudre les équations indiquées au $\$ 2.1$, il existe plusieurs approches, ce qui se traduit par un large choix de discrétisations en espace et en temps. Nous nous limiterons à la présentation d'une discrétisation en temps permettant d'utiliser des méthodes soit de différences finies, soit de volumes finis, soit d'éléments finis.

\section{A1.1 Techniques de décomposition des opérateurs ap-} pliquée à une équation d'advection-diffusion

L'idée de base est de dissocier la résolution de chaque opérateur (convection-diffusion-continuité) présent dans les équations de façon à choisir une méthode numérique bien adaptée à son comportement physique [34, 36, 37].

Cet algorithme sera introduit d'abord dans une équation d'advection-diffusion simple, puis sera appliqué au système complet d'équations à résoudre (Cf. \$2).

\section{A1.1.1 L'équation d'advection-diffusion}

Prenons l'équation d'advection-diffusion suivante :

$$
\begin{cases}\frac{\partial C}{\partial t}+u_{j} \frac{\partial C}{\partial x_{j}} & \\ =\frac{\partial}{\partial x_{j}}\left(K_{c} \frac{\partial C}{\partial x_{j}}\right)+S_{c} & \text { dans } \Omega \times[0, T] \\ K_{c} \frac{\partial C}{\partial n}=q_{d} & \text { sur } \Gamma_{n} \times[0, T] \\ C=C_{d} & \text { sur } \Gamma_{d} \times[0, T] \\ C\left(x_{i}, 0\right)=C_{0}\left(x_{i}\right) & \text { dans } \Omega,\end{cases}
$$

où $\Omega$ est un sous-ensemble borné ouvert régulier de $\mathbf{R}^{N}$ ( $N$ est la dimension de l'espace physique), $\Gamma$ est la limite de $\Omega$ (il est supposé suffisamment régulier, c'est-à-dire tel qu'il existe une normale presque partout). En outre, nous introduisons la partition :

$$
\Gamma=\Gamma_{n} \cup \Gamma_{d} \text { et } \Gamma_{n} \cup \Gamma_{d}=0
$$

$\left(u_{i}\right)_{i=1, N}: \Omega \times[0, T] \rightarrow \mathbf{R}^{N}$ est le champ de vitesse convective, supposé connu et satisfaisant l'équation de continuité :

$$
\partial u_{i} / \partial x_{i}=0
$$

$K_{c}$ est le coefficient de diffusivité associé à la quantité scalaire $C\left(x_{i}, t\right)$. ( $K_{c}$ peut varier dans l'espace et dans le temps, surtout lorsque l'écoulement est turbulent). Les valeurs données de $C$ (resp. de son flux) sur la frontière $\Gamma_{d}\left(\right.$ resp. $\Gamma_{n}$ ) sont notées $C_{d}$ (resp. $q_{d}$ ).
Soit $\Delta t>0$ le pas de temps et $t^{n}=n \Delta t$. L'approximation de $C$ au temps $t^{n}$ sera notée $C^{n}$. Supposons que $C^{n}$ soit connu au précédent pas de temps et décrivons le calcul de $C^{n+1}$. Suivant un algorithme conçu par Chorin [34], chaque pas de temps est subdivisé en deux sous-pas.

\section{A1.1.2 Étape de convection et méthode des caractéristiques}

Pour résoudre l'équation d'advection-diffusion, on utilise une approche lagrangienne. L'étape de convection consiste à calculer un champ convecté : $\bar{C}: \Omega \rightarrow \mathbf{R}$ qui permet de prendre en compte le terme de convection de l'équation d'advection-diffusion.

Pour n'importe quel champ $\left(\omega_{j}\right): \Omega \rightarrow \mathbf{R}^{N}$, on note $\Gamma_{-}\left(\omega_{j}\right)$ le sous-ensemble de $\Gamma$ défini par $^{4}$

$$
\Gamma_{-}\left(\omega_{j}\right)=\left\{\left(x_{i}\right)_{i=1 . N} \in \Gamma \mid \omega_{j} \cdot n_{j}\left(x_{i}\right)<0\right\}
$$

Supposons que pour le champ des vitesses $u_{j}$ on ait : $\Gamma_{-}(u j) \subseteq \Gamma_{d}$ et considérons le problème suivant.

Trouver $\zeta: \Omega \times] t^{n}, t^{n+1}[\rightarrow \mathbf{R}$ tel que :

$$
\left\{\begin{array}{l}
\left\{\frac{\partial \varepsilon}{\partial t}+u_{j} \frac{\partial}{\partial x_{j}} \xi\right\}=0 \quad \text { sur } \quad \Omega \times\left[t^{n}, t^{n+1}\right] \\
\xi\left(x_{j}, t^{n}\right)=C^{n}\left(x_{j}\right) \quad x_{j} \in \Omega \\
\xi=C_{d} \quad \operatorname{sur} \Gamma-\left(u_{j}\right) \times\left[t^{n}, t^{n+1}\right] .
\end{array}\right.
$$

On pose :

$$
\dot{C}\left(x_{j}\right)=\xi\left(x_{j}, t^{n+1}\right) .
$$

Il faut maintenant décrire le calcul de la quantité $\zeta\left(x_{j} t^{n+1}\right)$. Pour tous les points $\left(x_{j}\right)$ de $\Omega$, on introduit la courbe caractéristique $X_{x}:\left[t^{n}, t^{n+1}\right] \rightarrow \mathbf{R}^{N}$, satisfaisant :

$$
\left\{\begin{array}{l}
\frac{\mathrm{d} X_{j x}}{\mathrm{~d} t}(t)=u_{j}\left(X_{j x}\right) \quad t \in\left[t^{n}, t^{n+1}[,\right. \\
X_{j x}\left(t^{n+1}\right)=x_{j} .
\end{array}\right.
$$

On considère la restriction de $\zeta$ tout au long de la caractéristique, qui est définie de la manière suivante:

$$
\Xi(t)=\xi\left(X_{j x}, t\right) \quad t \in\left[t^{n}, t^{n+1}\right] .
$$

On dérive de (A1-4) que pour tout $t \in\left[t^{n}, t^{n+1}\right]$,

$$
\frac{\mathrm{d} \Xi}{\mathrm{d} t}(t)=\left(\frac{\partial \xi}{\partial t}+u_{j} \frac{\partial \xi}{\partial x_{j}}\right)\left(X_{j x}(t), t\right) .
$$

La quantité $\xi\left(x_{j} t^{n+1}\right)=\xi\left(X_{x j}\left(t^{n+1}\right), t^{n+1}\right)(\mathrm{Cf}$. (A1-4)) est calculée par intégration de l'équation (A1-6) de la manière suivante :

(i) $\mathrm{Si} X_{x j}\left(\left[t^{n}, t^{n+1}\right]\right) \subset \tilde{\Omega}$ Alors selon (A1-2) et (A1-3) on a :

$$
\tilde{C}\left(x_{j}\right)=C^{n}\left(X_{x j}\left(t^{n}\right)\right) .
$$

(4) Avec la convention d'Einstein : $a_{i} \cdot b_{i}=\sum_{i=1}^{N} a_{i} b_{i}$. 
(ii) Si $X_{x j}\left(\left[t^{n}, t^{n+1}\right]\right) \varnothing \bar{\Omega}$, alors on introduit le plus petit temps $\tau \in\left[t^{n}, t^{n+1}\right]$ tel que $X_{x j}\left(\left[\tau, t^{n+1}\right]\right) \subset \bar{\Omega}$. On a alors $X_{x j}(\tau) \in \Gamma_{-}\left(u_{j}\right)$ et on dérive de (A1-2) et (EA1-3) que :

$$
\tilde{C}\left(x_{j}\right)=C_{d}\left(X_{x j}(\tau), \tau\right) .
$$

N.B. : En pratique, pour le calcul de $\tilde{C}$ défini ci-dessus, la courbe caractéristique, solution de (A1-4), est approchée à l'aide d'une méthode de Runge-Kutta du second ordre.

\section{A1.1.3 Étape de diffusion}

A partir d'une discrétisation de type différences finies en temps du premier ordre implicite, le problème est maintenant de trouver $C^{n+1}$ à partir de :

$$
\left\{\begin{array}{l}
\frac{C^{n+1}-\tilde{C}}{\Delta t}-\frac{\partial}{\partial x_{j}}\left(K_{c} \frac{\partial C^{n+1}}{\partial x_{j}}\right)=S_{c} \\
\text { dans } \Omega \times[0, T] \\
K_{c} \frac{\partial C}{\partial n}=q_{d} \quad \text { sur } \Gamma_{n} \times[0, T] \\
C=C_{d} \quad \text { sur } \Gamma_{d} \times[0, T] .
\end{array}\right.
$$

A1.1.4 Quelques propriétés de ce système

(1) Le terme $\left(C^{n+1}-\dot{C}\right) / \Delta t$ est une approximation du premier ordre du terme de convection

$$
\frac{\partial C}{\partial t}+u_{j} \frac{\partial C}{\partial x_{j}}
$$

au point $\left(x_{j} t^{n+1}\right)$. Cette assertion est démontrée dans [36]. En conséquence, le système est consistant.

(2) On obtient la convergence et la stabilité du système à partir du résultat suivant dû à Pironneau [36] :

Supposons que le champ de convection soit suffisamment régulier. Dans les cas où $\Gamma_{n}=0$ et pour un coefficient constant $K_{c}$, le système précédent est inconditionnellement stable au sens de la norme $L^{2}{ }^{5}$ En outre, on a la majoration d'erreur suivante :

$$
\begin{aligned}
\left(\| C^{n+1}-C\left(., t^{n+1}\right)\right. & \left\|_{0}^{2}+v \Delta t\right\| \frac{\partial}{\partial x_{j}}\left(C^{n+1}\right. \\
& \left.\left.-C\left(., t^{n+1}\right)\right) \|_{0}^{2}\right)^{1 / 2} \leq A \Delta t,
\end{aligned}
$$

où $A$ est une constante ne dépendant que de $\Omega$. Pour une démonstration de ce résultat, se reporter à Pironneau [36] ou Chabard et coll. [37].

\section{A1.2 Application du système précédent aux équations de Navier-Stokes}

La méthode décrite ci-dessus dite méthode à pas fractionnaires peut être appliquée de la manière suivante aux

( $\left.{ }^{5}\right) L^{2}$ est l'espace des fonctions de carrés sommables définies dans $\Omega$. Dans cet espace, on peut définir la norme suivante : $\|f\|_{0}^{2}=\int_{\Omega} f^{2} \mathrm{~d} \Omega$ équations de Navier-Stokes (2), (3), (4) écrites en utilisant la moyenne de Reynolds et données au $\$ 2.1$.

\section{A1.2.1 Étape de convection}

Dans le calcul du champ des vitesses au temps $t^{n+1}$, le champ de convection sera la vitesse au pas de temps précédent. En conséquence, étant donné $\left(u_{j}\right)^{n}$, on définit une vitesse $\left(v_{j}\right)$ - en reprenant les formules (A1-7) et (A1-8) pour chacune des composantes de vitesse - en prenant successivement $C=u_{1}, u_{2}$ et $u_{3}$.

\section{A1.2.2 Étape de Stokes}

On calcule $\left(u_{j}\right)^{n+1}: \Omega \rightarrow \mathbf{R}^{N}$ et $p^{n+1}: \Omega \rightarrow \mathbf{R}$ solutions de :

$$
\begin{cases}\frac{u_{i}^{n+1}-\tilde{u}_{i}}{\Delta t}-\frac{\partial}{\partial x_{j}}\left(\mu_{e}^{n} \frac{\partial}{\partial x j} u_{i}^{n+1}\right) & \\ =-\frac{\partial}{\partial x_{i}} p^{n+1}+S_{i}^{n+1} & \text { dans } \Omega \\ \partial u_{i}^{n+} / \partial x_{i}=0, & \text { dans } \Omega \\ \partial u_{i} / \partial x_{i}=0 & \operatorname{sur} \Gamma_{d} \\ \left(u_{i}\right)^{n+1}=\left(u_{i}\right) d\left(., t^{n+1}\right) & \operatorname{sur} \Gamma_{n}, \\ \mu_{\varepsilon}^{n} \frac{\partial u_{i}^{n+1}}{\partial n}=\sigma_{i}\left(., t^{n+1}\right) & \end{cases}
$$

où :

$$
\mu_{e}^{n}=\mu+\mu_{t}^{n},
$$

$\mu_{t}^{n}$ est donné par le modèle de turbulence, et $S_{i}^{n+1}$ est le terme de flottabilité.

N.B. : On a le même type de majoration d'erreur que pour l'équation scalaire d'advection-diffusion.

\section{A1.3 Discrétisation spatiale}

Selon le code, il existe deux types de discrétisation.

\section{A1.3.1 Code ESTET}

C'est un code 3D en différences finies. Les équations sont discrétisées sur une grille orthogonale, avec des coordonnées cartésiennes ou cylindriques (dans les deux cas les composantes cartésiennes de la vitesse sont calculées), cf. [14].

La vitesse $u_{j}$, la température $T$, l'énergie cinétique turbulente $k$ et le taux de dissipation $\varepsilon$ sont calculés aux nouds du maillage alors que la pression est discrétisée sur une grille décalée dont les points sont placés au centre d'une cellule de vitesse définie par 8 points.

Pour obtenir une meilleure description des géométries complexes 3D, des cellules limites incomplètes sont définies selon les divers tétraèdres et prismes qui peuvent être construits en supprimant un ou plusieurs coins d'une cellule rectangulaire définie par 8 points. Ainsi, on parvient à une plus grande précision dans la description des frontières solides. 
L'algorithme à pas fractionnaire décrit ci-dessus peut être entièrement vectorisé lorsqu'il est appliqué à des maillages rectangulaires orthogonaux. En conséquence, on peut parvenir à des coûts d'utilisation d'unité centrale très intéressants sur des supercalculateurs vectoriels tels que les CRAY (à l'heure actuelle, environ $\backslash \operatorname{mbox}\{0,1 \mathrm{~s}\}$ par pas de temps et pour 1000 nœuds pour des calculs 3D de thermohydraulique, le temps d'utilisation de l'unité centrale augmentant de façon quasi linéaire en fonction du nombre de nouds).

Comme il est cependant difficile de décrire précisément des géométries très complexes en utilisant des maillages orthogonaux (même avec des cellules incomplètes) une version curviligne du code ESTET a été mise au point. Elle sert surtout à calculer les stratifications thermiques dans des écoulements en conduites qui interviennent dans certaines analyses thermohydrauliques (Cf. [14]). Pour des géométries plus complexes, le code N3S, qui utilise une discrétisation en éléments finis, a été développé à EDF.

\section{A1.3.2 Code N3S}

Il est désormais reconnu que les méthodes d'éléments finis permettent de décrire précisément des géométries complexes et d'affiner localement les maillages, c'est d'ailleurs ce qui les rend très intéressantes.

N3S est un code généraliste 2D et 3D. La discrétisation spatiale peut utiliser soit des tétraèdres ou des triangles P1--P2 ou P1--isoP2.

Pour résoudre les équations de Navier-Stokes pour des fluides incompressibles, le choix d'un élément fini est limité par ce qu'on appelle la condition inf-sup. Cette condition assure l'existence et l'unicité de la solution du problème de Stokes et empêche des modes parasites en pression. Tous les éléments utilisés par le code N3S satisfont cette condition.

Après une phase de validation et d'optimisation, le code N3S en éléments finis est aujourd'hui largement utilisé pour des études industrielles. Le temps d'utilisation d'unité centrale est légèrement plus important qu'avec le code ESTET en différences finies (à l'heure actuelle, Imbox $\{0,3 \mathrm{~s}\}$ par pas de temps pour 1000 nouds pour un calcul 3D de thermohydraulique ; le temps d'utilisation de l'unité centrale augmentant de façon quasi linéaire en fonction du nombre de nœuds), mais ce surcoût est compensé par l'économie en nombre de points de maillage que permet l'utilisation de maillages non structurés en tétraèdres.

\section{A1.4 Algorithme numérique pour le problème de Stokes}

\section{A1.4.1 Code ESTET}

Dans ce code, la diffusion des composantes de vitesse est traitée au même pas que celles des quantités scalaires. L'équation de pression-continuité peut s'écrire :

$$
\left\{\begin{array}{l}
u_{i}^{n+1}+\Delta t \frac{\partial p^{n+1}}{\partial x_{i}}=\Delta t S_{i}^{n+1}+\tilde{u}_{i} \\
\partial u_{i}^{n+1} / \partial x_{i}=0,
\end{array}\right.
$$

où $\tilde{\tilde{u}}_{i}$ est obtenu par convection et diffusion de $u_{i}^{n}$.
A partir de l'opérateur de divergence appliqué à l'équation de conservation de la quantité de mouvement, on peut obtenir pour la pression ce qu'on appelle l'équation de Poisson qui s'écrit :

$$
\Delta p^{n+1}=\frac{\partial S_{i}^{n+1}}{\partial x_{i}}+\frac{\partial \tilde{u}_{i}}{\partial x_{i}} .
$$

On résoud cette équation en prenant des conditions aux limites de Neumann homogènes sur $p^{n+1}\left(\partial p^{n+1} / \partial n=0\right)$.

On obtient alors $\left(u_{i}\right)^{n+1}$ en résolvant la première équation dans (??).

\section{Al.4.2 Code N3S}

Dans ce code, la diffusion des composantes de vitesse est traitée au même pas que la continuité. Dans la méthode des éléments finis, la discrétisation en espace se fait à partir de la formulation variationnelle des équations. Lorsque cette méthode est appliquée au problème de Stokes et après discrétisation, on obtient la forme matricielle suivante :

$$
\left[\begin{array}{ll}
A & B_{t} \\
B & 0
\end{array}\right]\left[\begin{array}{l}
U^{n+1} \\
P^{n+1}
\end{array}\right]=\left[\begin{array}{c}
S^{n+1} \\
0
\end{array}\right]
$$

avec $A=M+\Delta t D$.

$M$ est la matrice de la masse, $D$ la matrice de la diffusion, $B$ et $B^{t}$ sont les matrices associées respectivement aux opérateurs de divergence et de gradient. $U^{n+1}$ et $P^{n+1}$ désignent les vecteurs de vitesse et de pression des valeurs nodales.

A partir de (A1-14) on obtient l'équation de pression discrétisée :

$$
\left(B A^{-1} B^{\prime}\right) P^{n+1}=-B A^{-1} S^{n+1},
$$

mais comme $A$ \# Id, il n'est pas réaliste de calculer et de stocker la matrice $B A^{-1} B^{\prime}$ (il s'agit d'une matrice pleine et son inversion exigerait beaucoup de temps d'unité centrale).

L'astuce consiste à résoudre $(\mathrm{Al}-15)$ avec un algorithme de gradient. C'est ce qu'on appelle l'algorithme d'Uzawa dont le principe est le suivant (Cf. 33) :

$$
\begin{aligned}
& \text { - étant donné } P_{m} \text {, } \\
& \text { - calculer } U_{m} \text { en résolvant : } A U_{m}=S-B P_{m} \\
& \text { - calculer le résidu du système }(\mathrm{A} 1-15): R_{m}=B U_{m} \\
& \text { - modifier la pression : } P_{m+1}=P_{m}-\rho R_{m} \\
& \text { - procéder par itérations jusqu'à : }\left\|R_{m}\right\|<\varepsilon
\end{aligned}
$$

N.B. :

- Le principal avantage de cet algorithme est d'éviter le calcul de $B A^{-1} B^{\prime}$.

- Lorsqu'on utilise des conditions aux limites simples, on peut calculer $U_{m}$ en dissociant les équations pour chacune des composantes de vitesse.

- On peut accélérer l'algorithme en combinant algorithme de gradient conjugué et techniques de préconditionnement (avec un bon préconditionnement, on obtient une diminution du résidu de 6 ordres de grandeurs en moins de 20 itérations [22]). 
- On peut satisfaire globalement la contrainte d'incompressibilité dans le domaine, aussi précisément qu'on le veut, parce que les itérations sont pilotées par $B U_{m}$ qui est l'approximation de $\operatorname{div} \mathbf{u}=\partial u_{i} / \partial x_{i}$

\section{Annexe 2 - Méthodes numériques utilisées dans le système TRIO de simulation d'écoulements 3D}

Le système TRIO développé par le CEA permet de simuler des écoulements 3D dans des situations industrielles. Il intègre les différents modules nécessaires pour simuler des écoulements dans des géométries complexes et pour en interpréter les résultats. Un préprocesseur génère le maillage et permet de définir les conditions aux limites et tous les modèles physiques nécessaires pour une simulation d'écoulements. Deux solveurs sont disponibles, qui font appel aux deux principales méthodes disponibles en mécanique des fluides numérique : la méthode des éléments finis et la méthode des volumes finis. La visualisation et le post-traitement se font à l'aide d'outils courants.

Les équations de conservation locale et les équations de transport pour les grandeurs turbulentes $k$ et $\varepsilon$, décrites au $\S 2$, sont résolues grâce à l'une des techniques numériques suivantes :

- la méthode des volumes finis,

- la méthode des éléments finis.

\section{A2.1 Méthode des volumes finis}

* Discrétisation spatiale dérivée d'équations de bilan macroscopique appliquées à des volumes de contrôle. Les variables sont situées sur un maillage décalé.

* Schéma numérique pour les termes de convection. Deux schémas sont appliqués :

- Schéma DonnorCell : approximation du premier ordre de la convection. Ce système est économique et robuste mais introduit une diffusion numérique lorsque l'écoulement est incliné par rapport aux lignes de maillage.

- Schéma QUICK filtré : schéma du troisième ordre utilisant une approximation quadratique sur trois points du scalaire transporté. Les trois points sont choisis de la manière suivante : deux vers l'amont et un vers l'aval. Un filtre empêche les oscillations non physiques.

\section{A2.2 Méthode des éléments finis}

Elle se fonde sur la méthode proposée par Gresho et coll. La discrétisation spatiale par éléments finis est effectuée selon la méthode de Galerkin, avec un élément triangulaire ou quadrilatéral bilinéaire en $2 \mathrm{D}$ et un élément prismatique ou cubique trilinéaire en $3 \mathrm{D}$.

Pour améliorer le rapport coût-efficacité, on utilise une quadrature réduite ( 1 point) avec la correction Hourglass. Des corrections supplémentaires ont été mises au point pour traiter certains écoulements turbulents. On calcule la partie sous-intégrée et la correction Hourglass avec la diffusivité moyenne. Un terme de correction tient compte des gradients de diffusivité qui peuvent être importants dans des écoulements turbulents.

\section{A2.3 Discrétisation en temps et algorithme de résolution}

Les deux modules résolvent les équations instationnaires. On utilise un schéma semi-implicite dans lequel les termes de transport sont écrits de façon explicite, les seuls termes implicites étant :

- l'équation de continuité et le gradient de pression dans l'équation de conservation de la quantité de mouvement, - la résistance hydraulique lorsqu'on rencontre de grandes variations de ce terme.

La manipulation algébrique des équations discrétisées permet de dériver un système algébrique pour les inconnues de pression.

Des algorithmes ont été mis au point qui réduisent les coûts de traitement informatique en faisant appel à la symétrie de la matrice et à ses propriétés d'invariance en temps.

\section{A2.4 Applications}

Ce logiciel de thermohydraulique couvre déjà une large panoplie d'applications :

- ventilation et écoulements autour de bâtiments,

- dispersion des polluants dans l'atmosphère,

- hydrogéologie et transport des polluants,

- échangeurs de chaleur,

- écoulements irréguliers et oscillants,

- refroidissement des moteurs de voitures, ventilation des voitures,

- générateurs de vapeur dans les REP.

Le système TRIO est décrit plus en détail dans [38, 40].

\section{Références}

[1] Launder B.E. et SPALding D.B. - The numerical computation of turbulent flows, Comput. Meth. Appl. Mech. Engrg. 3 (1974)

[2] RoDI W. - Turbulence Models and their Application in Hydraulics, AIRH, Delft, Pays-Bas (1980).

[3] Gibson M.M. et Launder K. (1976), - ASME J. Heat Transfer 98, 81 .

[4] VIOLLET P.L. - The modelling of turbulent recirculating flows for the purpose of reactor thermal-hydraulic analysis, Nucl. Engrg. Des. 99, 365-377 (1987).

[5] VIOLLeT P.L. - On the numerical modelling of stratified flows, In : Physical Processes in Estuaries, (eds. Dronkers and Van Leussen) Springer Verlag, pp. 257-277 (1988).

[6] KHoudu M. - Macrosimulation de turbulence homogène en présence de cisaillements et de gradients thermiques: application aux modèles de fermeture en un point. Thèse, Ecole Centrale de Lyon, décembre 1988.

[7] Hutton A.G. et SZEPURA R.T. - Turbulent flow and heat transfer in a pipe expansion : a comparison of current models of turbulence, Rapport CEGB TPRD/B/0926/R87, décembre 1987. 
[8] BARON F. - Data and conclusion of the 12th meeting of IAHR Working Group on Refined Modelling of Flows, EDF Rap-HE44/88.28, juillet 1988.

[9] WILKES N.S. - Working Group on Refined Modelling of Flows, Eight Meeting, Harwell, avril 1984, Final Report on test problem, AERE-R 12493.

[10] Normandin M. - Etude expérimentale de l'écoulement turbulent dans une cavité profonde, Thèse Université Scientifique et Médicale et Institut National Polytechnique de Grenoble.

[11] Vidil R., GRAND D. et LeRoux F. - Interaction of recirculation and stable stratification in a rectangular cavity filled with sodium, Nucl. Engrg. Des. 105, 312-332 (1988).

[12] Grand D., Meyer D., Viollet P.L. et Lacroix C. Comparison between experimental and numerical studies on the prediction of thermal stratification in a rectangular cavity, Proc. Int. Symp. on Buoyant Flows (ed. Pr. G. Noutsopoulos) Athène, Grèce, 1-5 septembre 1986.

[13] SIMPSON J.E. et BRITTER. - Experiments on the dynamics of the front of a gravity current. Proc. 2nd Int. Symp. on Stratified Flows, Trondheim, pp. 174-183, Tapir (1980).

[14] Gabillard M. et Viollet P.L. - The three-dimensional computation of secondary flow and density currents in a curved pipe, Proc. 3rd Int. Symp. on Refined Flow Modelling and Turbulence Measurements, Tokyo, juillet 1988.

[15] VIOLLet P.L. - Observation and numerical modeling of density currents resulting from thermal transients in a non rectilinear pipe, J. Hydr. Res., 25 (2), 235-255 (1987).

[16] TOLY J.A. et LE GoFF J. - Thermal stratifications in pipes : NAJET sodium tests on REYCUR calculations, ASME 84WA/HT-113.

[17] TENCHINE D. et GRAND D. - Onset of natural circulation in a sodium loop, 4th Int. Meeting on Nuclear Reactor Thermalhydraulics, Karlsruhe, 10-13 octobre 1989.

[18] SChumanN U. - The countergradient heat flux in turbulente stratified flows, Nuc. Engrg. Des., 100, 255-262 (1987).

[19] Launder B.E., ReECE G.J. et Rodi W. - Progress in the development of a Reynolds-stress turbulence closure, J. Fluid Mech., 68 (3), 537-566 (1975).

[20] LAUNDER B.E. - Second moment closure : methodology and practice, In : Turbulence models and their applications, Eyrolles, Paris, pp. 3-147 (1989).

[21] CCHUNG M.K. et SUNG H.J. - Four-equation turbulence model for prediction of the turbulent boundary layer affected by buoyancy force over a flat plate, Int. J. Heat \& Mass Transfer, 27, 2387-2395 (1984).

[22] KANNICHE M., BARON F. et VIOLLet P.L. - Second moment closure prediction of recirculating flow and of countergradient heat flux in stratified flow, 7th Int. Symp. on Turbulent Shear Flows, Stanford, 21-23 août 1989.

[23] Hossain M.S. et Rodi W. - Mathematical modelling of vertical mixing in stratified channel flow, 2nd Int. Symp. on Stratified Flows, Trondheim, pp. 280-290, Tapir (1980).

[24] HuANg P.G. et Leschziner M.A. - Stabilization of recirculating flows computations performed with second-moment closure and third other discretization, Proc. 5th Int. Symp. on Turbulent Shear Flows, Ithaca, août 1985, Springer Verlag.
[25] KANNICHE M., BARON F. et Viollet P.L. - Incompressible flow calculations with a full Reynolds-stress and turbulent fluxes transport model, Proc. 3rd Int. Symp. on Refined Flows Modelling and Turbulence Measurements, Tokyo, juillet 1988.

[26] Mompean G., PhD. - Modélisation des écoulements turbulents avec transferts thermiques en convection mixte, Thèse, CEA \& Ecole Centrale de Lyon, juin 1989.

[27] Peniguel C. et Lacroix C. - Résultats non publiés (1988).

[28] GRAND D. et Vernier Ph. - Forced convection heat transfer in liquid metals, Turbulent Forced Convection in Channels and Bundles (ed. Kabaç and Spalding), Hemisphere (1979).

[29] PIMONT V. - Etude et modélisation d'écoulements turbulents de métaux liquides, Thèse de Docteur Ingénieur, Ecole Centrale des Arts et Manufactures.

[30] EYLER L.L. - Turbulent structure measurements and thermal transport modeling in liquid metal, Thèse de $\mathrm{PhD}$, Université de Purdue.

[31] HinZE J.O. - Turbulence, McGrawHill, $2^{\circ}$ éd., pp. 175-315 (1975).

[32] Chabard J.P., Jami A. et LalanNe P. - Finite element solution of turbulent flows, EDF Rapport Ref. HE41/88.35.

[33] Cahouet J. et ChaBARD J.P. - Some fast 3D finite element solvers for generalized Stokes Problem, Int. J. Numer. Methods in Fluids, vol. 8, 869-895 (1988).

[34] Chabard J.P. et Daubert O. - A 3D finite element code for industrial applications, EDF Rapport Ref. HE41/88.17 et Proc. Conf. on Numerical Methods for Fluids Dynamics, Oxford, R.U. (1988).

[35] CHORIN A.J. - Numerical Solution of incompressible flow problem, SIAM J. Numer. Anal., 2 (1968).

[36] Pironneau O. - Méthode des Eléments Finis, Masson (1988).

[37] Chabard J.P., Metivet B., Рot G. et Thomas B. - An efficient finite element method for the computation of $3 D$ turbulent incompresible flows, Int. J. Numer. Meth. Fluids, à paraitre.

[38] Benque J.P., Daubert O., Goussebaile J. et Hauguel A. - Splitting up techniques for computations of industrial flows, In : Vistas in Applied Mathematics (ed. A.V. Balakrishman), Springer (1986).

[39] Hoffman A., Magnaud J.P., Grand D. et Villand M. Recent developments in the numerical prediction of thermal hydraulics, Int. Topical Meeting on Advances in Reactor Physics, Paris, 27-30 avril 1987.

[40] Grand D., Coulon N., Magnaud J.P. et Villand M. Computation of flows with distributed resistance and heat sources (1988).

[41] Rouzaud Ph., Magnaud J.P., Bertrand G., Thiry J.M., VILLAND M. et GRAND D. - Numerical simulation using TRIO of multidimension flows with heat transfer, Advances in Nuclear Engineering Computation and Radiation Shrelding, Santa Fe, 9-13 avril 1989.

[42] POPE S.B. - The statistical theory of turbulent flames, Philos. trans. R. Soc. London A 291, 529-568 (1979). 